Check for updates

Cite this: RSC Adv., 2018, 8, 5864

\title{
Hyaluronan-based graft copolymers bearing aggregation-induced emission fluorogens $\uparrow$
}

\author{
Andrea Cappelli, (D) *a Marco Paolino, (D) a Annalisa Reale, ${ }^{a}$ Vincenzo Razzano, ${ }^{a}$ \\ Giorgio Grisci, ${ }^{a}$ Germano Giuliani, ${ }^{a}$ Alessandro Donati, ${ }^{a}$ Claudia Bonechi, ${ }^{a}$ \\ Stefania Lamponi, ${ }^{a}$ Raniero Mendichi, ${ }^{b}$ Salvatore Battiato, (D) ${ }^{c}$ Filippo Samperi, ${ }^{c}$ \\ Francesco Makovec, ${ }^{d}$ Mariano Licciardi, (D) ${ }^{\text {ef }}$ Lorenzo Depau (DD ${ }^{g}$ and Chiara Botta (DD ${ }^{b}$
}

In order to develop a technology platform based on two natural compounds from biorenewable resources, a short series of hyaluronan (HA) copolymers grafted with propargylated ferulic acid (HA-FA-Pg) were designed and synthesized to show different grafting degree values and their optical properties were characterized in comparison with reference compounds containing the same ferulate fluorophore. Interestingly, these studies revealed that the ferulate fluorophore was quite sensitive to the restriction of intramolecular motion and its introduction into the rigid HA backbone, as in HA-FA-Pg graft copolymers, led to higher photoluminescence quantum yield values than those obtained with the isolated fluorophore. Thus, the propargyl groups of HA-FA-Pg derivatives were exploited in the coupling with oleic acid through a biocompatible nona(ethylene glycol) spacer as an example of the possible applications of this technology platform. The resulting HA-FA-NEG-OA materials showed self-assembling capabilities in aqueous environment. Furthermore, HA-FA-NEG-OA derivatives have been shown to interact with phospholipid bilayers both in liposomes and living cells, retaining their fluorogenic properties and showing a high degree of cytocompatibility and for this reason they were proposed as potential biocompatible self-assembled aggregates forming new materials for biomedical applications.

Received 17th November 2017 Accepted 26th January 2018

DOI: $10.1039 / c 7 r a 12543 g$

rsc.li/rsc-advances

\section{Introduction}

The hydroxycinnamic derivative ferulic acid (FA, 4-hydroxy-3methoxycinnamic acid) is largely distributed in the plant cellular wall, ${ }^{1}$ where it is linked by means of an ester bond to primary alcohol groups of arabinose side chains of arabinoxylans. The FA residues inserted in the cell wall of

${ }^{a}$ Dipartimento di Biotecnologie, Chimica e Farmacia and European Research Centre for Drug Discovery and Development, Università degli Studi di Siena, Via A. Moro 2, 53100 Siena, Italy. E-mail: andrea.cappelli@unisi.it; Tel: +390577234320

${ }^{b}$ Istituto per lo Studio delle Macromolecole (CNR), Via A. Corti 12, 20133 Milano, Italy 'Istituto per i Polimeri, Compositi e Biomateriali (IPCB) U.O.S. di Catania, CNR, Via Gaifami 18, 95126 Catania, Italy

${ }^{d}$ Rottapharm Biotech, Via Valosa di Sopra 9, 20900 Monza, Italy

${ }^{e}$ Dipartimento di Scienze e Tecnologie Biologiche, Chimiche e Farmaceutiche (STEBICEF), Università degli Studi di Palermo, Via Archirafi 32, 90123 Palermo, Italy ${ }^{f}$ Mediterranean Center for Human Health Advanced Biotechnologies (CHAB), University of Palermo, Italy

${ }^{g}$ Dipartimento di Biotecnologie Mediche, Università degli Studi di Siena, Via A. Moro 2, 53100 Siena, Italy

$\dagger$ Electronic supplementary information (ESI) available: Synthetic procedures for the preparation of azide 3 and of model compounds 5 and FA-NEG-OA; ${ }^{1} \mathrm{H}$ NMR spectrum of HA-FA-NEG-OA-8 compared with that of HA-FA-NEG-OA-4; ${ }^{13} \mathrm{C}$ NMR spectrum obtained with HA-FA-NEG-OA-4 derivative compared with those obtained with HA, HA-FA-Pg-3F, and model compound FA-NEG-OA; absorption spectra of compounds 4 and 6. See DOI: $10.1039 / \mathrm{c} 7 \mathrm{ra12543g}$ polysaccharides play the crucial role of cross-linking polysaccharides and proteins during cell wall synthesis. ${ }^{1-4}$ Furthermore, FA residues can be transformed into a large variety of dehydrodiferulate dimers capable of bridging different polysaccharide chains by peroxidase-mediated oxidative coupling (or dimerization and photoisomerism by UV light). ${ }^{1-4}$ FA also possesses interesting fluorescence properties, with absorption/ emission features affected by both solvent polarity and $\mathrm{pH} .{ }^{5} \mathbf{F A}$ has been observed to be an inhibitor of beta amyloid pathological aggregation ${ }^{6}$ and has been incorporated into biodegradable polymers as a pendant group to enhance antioxidant properties for tissue engineering applications..$^{7-9}$ Polymers containing FA in the main chain have also been proposed to obtain materials for sensing and/or imaging applications. ${ }^{10-16}$ Aggregation induced emission (AIE) in biocompatible fluorophores have gained much attention in recent years since AIE compounds, being weakly emissive or non-emissive in solution due to free intramolecular motions, become good emitters upon aggregation and therefore represent a class of excellent fluorescent bioprobes. ${ }^{17}$

The glycosaminoglycan hyaluronic acid (HA, hyaluronan) plays important roles in human body. Among them, HA forms a pericellular coat playing a pivotal role in the early stages of cell adhesion ${ }^{18}$ by interacting with CD44 receptor. ${ }^{19,20}$ This receptor is an ubiquitous protein possessing an extracellular portion 
called hyaluronan binding domain and has been described to be expressed in high density in tumor tissues. ${ }^{21}$

In our previous studies, the HA backbone was grafted with variable densities of FA residues to afford HA-FA graft copolymers in the aim of combining the intriguing properties of the two natural compounds. ${ }^{22,23}$ Furthermore, a tri-component polymer brush was designed by functionalization of a polybenzofulvene derivative with nona(ethylene glycol) (NEG) side chains terminated with low molecular weight hyaluronic acid macromolecules. ${ }^{\mathbf{2 4 2 5}}$ This material was prepared by means of a convergent approach employing a copper(I)-catalyzed azidealkyne 1,3-dipolar cycloaddition (CuAAC) of the suitable polybenzofulvene derivative (the azide component) with the hyaluronan derivative HA-FA-Pg (Fig. 1) bearing propargyl groups bound to HA backbone through FA fluorophores. ${ }^{24}$

In order to develop a technology platform based on two natural compounds from biorenewable resources, we prepared a short series of hyaluronan-based graft copolymers (HA-FA-Pg) showing different grafting degree (GD) values and we studied their optical properties. In the attempt to demonstrate the usefulness of the technology platform, the propargyl groups of HA-FA-Pg derivatives were exploited in the CuAAC coupling with alpha-azido-omega-oleic amide nona(ethylene glycol) (compound 3, azido-NEG-OA) to obtain amphiphilic HA derivatives (i.e. HA-FA-NEG-OA). The obtained HA-FA-NEGOA showed potential self-assembling abilities in aqueous environment and for this reason was herein exhaustively characterized and proposed as biocompatible material with high potentiality in biomedical applications (i.e. in drug delivery).

\section{Experimental section}

\section{Synthesis and characterization}

All reagents and solvents were purchased from Sigma-Aldrich and were used as received, with the exceptions noted. Merck TLC aluminum sheets, silica gel $60 \mathrm{~F}_{254}$ were used for TLC. NMR spectra were recorded with either a Bruker DRX-400 Avance, a Bruker DRX-500 AVANCE, a Bruker AMX-600 Avance, or a Bruker Avance 900 spectrometer, working at $900.13 \mathrm{MHz}$ frequency and equipped with a cryogenically cooled probe in the indicated solvents (TMS as internal standard).

\section{General procedure for the preparation of HA-FA-Pg graft copolymers}

Into a two-necked round-bottomed $50 \mathrm{~mL}$ flask, a mixture of low molecular weight HA (8700 Da, Biophil Italia SpA, see the amounts in Table 1) in formamide $(10 \mathrm{~mL})$ was heated from 23

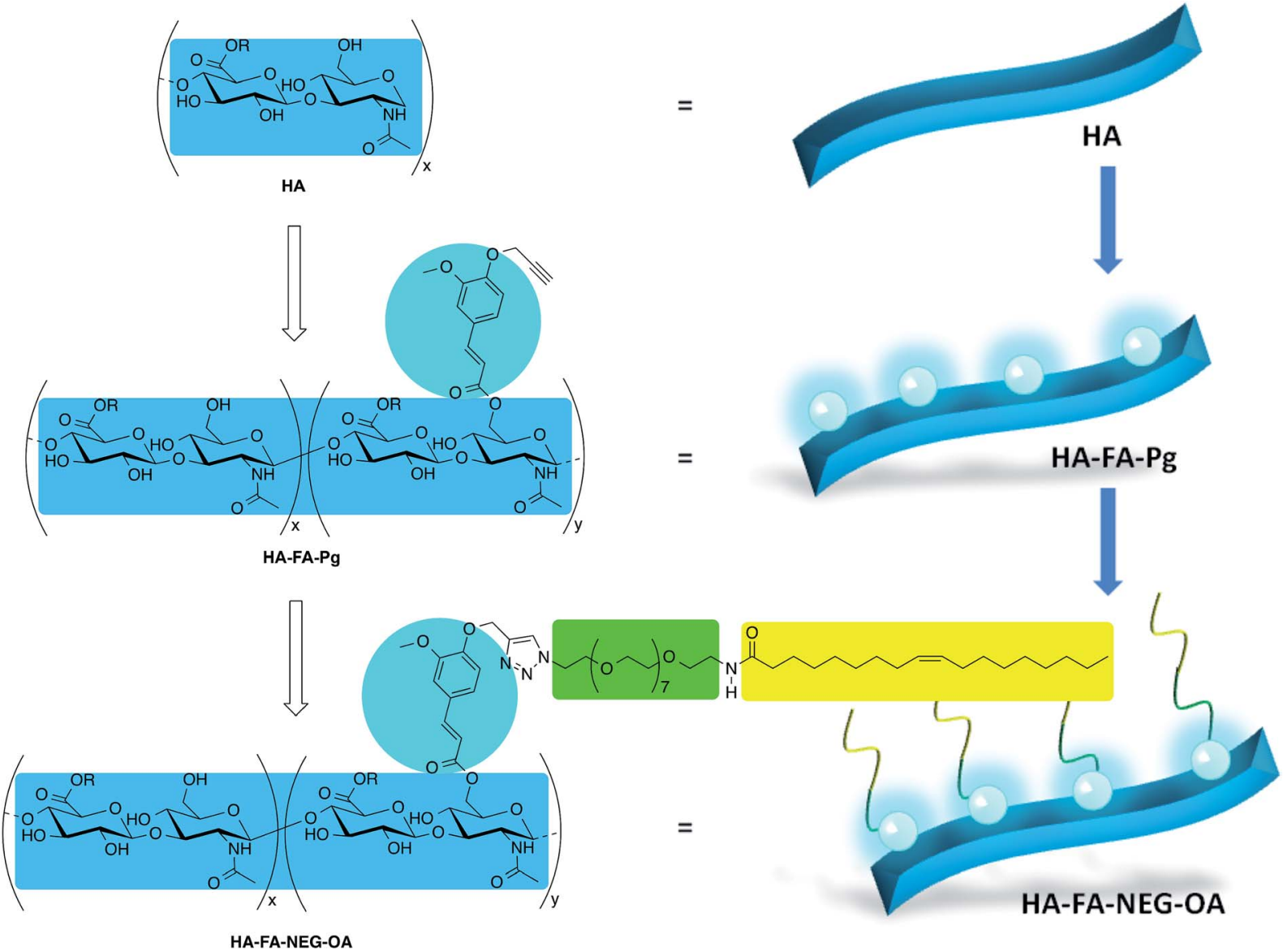

Fig. 1 Design of multi-component material HA-FA-NEG-OA based on hyaluronic acid (HA, light blue), ferulic acid (FA, cyan), nona(ethylene glycol) (NEG, green), and oleic acid (OA, yellow). Substituents: $\mathrm{R}=\mathrm{H}$ or $\mathrm{C}_{2} \mathrm{H}_{5}$. 
Table 1 Reaction parameters in the functionalization of HA polymer to HA-FA-Pg graft copolymers with imidazolide 1

\begin{tabular}{llllllc}
\hline Entry & Copolymer & HA $(\mathrm{g})$ & HA $(\mathrm{mmol})$ & $\mathbf{1 / H A}$ ratio $(\%)$ & Grafting degree $^{a}(\%)$ \\
\hline 1 & HA-FA-Pg-01 & 0.50 & 1.32 & 25 & 20 & Convers. $^{b}(\%)$ \\
2 & HA-FA-Pg-02 & 0.50 & 1.32 & 25 & 18 & 72 \\
3 & HA-FA-Pg-03 & 0.50 & 1.32 & 25 & 20 & 33 \\
4 & HA-FA-Pg-1F & 0.39 & 1.03 & 50 & 35 & 66 \\
5 & HA-FA-Pg-2F & 0.50 & 1.32 & 50 & 20 & 70 \\
6 & HA-FA-Pg-3F & 1.0 & 2.64 & 25 & 10 & 80 \\
7 & HA-FA-Pg-4F & 1.0 & 2.64 & 12.5 &
\end{tabular}

${ }^{a}$ A rough estimate of the grafting degree was made by ${ }^{1} \mathrm{H}$ NMR spectroscopy after hydrolysis with NaOD in $\mathrm{D}_{2} \mathrm{O}$ as described in ref. $22 .{ }^{b}$ The conversion into ferulate was calculated from the substitution degree and stoichiometric ratio 1/HA. ${ }^{c}$ See ref. 24.

to $50{ }^{\circ} \mathrm{C}$ till the complete dissolution was obtained. The resulting solution was then cooled to room temperature and triethylamine (TEA, $0.37 \mathrm{~mL}, 2.66 \mathrm{mmol}$ ) and $\mathbf{1}^{24}$ (see the amounts in Table 1) were added in sequence. After stirring the reaction mixture overnight at room temperature, a $5 \% \mathrm{NaCl}$ solution $(5.0 \mathrm{~mL})$ was added and the resulting mixture was stirred at room temperature for additional 10-15 $\mathrm{min}$. The expected graft copolymer derivative was obtained by treatment of the mixture with acetone $(40 \mathrm{~mL})$ and purified by washing four times with the same solvent. The final solid was dried under reduced pressure to afford the expected HA-FA-Pg graft copolymer as a white solid. ${ }^{1} \mathrm{H}$ NMR $\left(600 \mathrm{MHz}, \mathrm{D}_{2} \mathrm{O}\right)$ : see Fig. $3 ;{ }^{13} \mathrm{C}$ NMR (125 MHz, $\mathrm{D}_{2} \mathrm{O}$ ): see Fig. 4.

\section{Synthesis of HA-FA-NEG-OA-4 material}

Under an inert atmosphere, a $10 \mathrm{~mL}$ flask was charged with tertbutanol $(2.0 \mathrm{~mL})$, water $(2.0 \mathrm{~mL})$, and a solution of $\mathrm{CuSO}_{4}$ pentahydrate $(12.5 \mathrm{mg}, 0.050 \mathrm{mmol})$ in $0.50 \mathrm{~mL}$ of water. A $1 \mathrm{M}$ solution of sodium ascorbate in water $(0.50 \mathrm{~mL})$ was then added and $1.0 \mathrm{~mL}$ of the resulting mixture was used as the catalyst. A mixture of 3 (37 mg, $0.0526 \mathrm{mmol}$ ) and HA-FA-Pg-3F graft copolymer $(100 \mathrm{mg})$ in water $(5.0 \mathrm{~mL})$ was treated with the catalyst solution $(1.0 \mathrm{~mL})$ and the reaction mixture was stirred at room temperature for $4 \mathrm{~h}$ and then treated with QUADRASIL MP (200 mg). After filtration the solution was concentrated under reduced pressure. Purification of the residue by washing with acetone gave HA-FA-NEG-OA-4 material, which was dried under reduced pressure to obtain as a light brown glassy solid (75 mg). ${ }^{1} \mathrm{H}$ NMR (500 MHz, D 2 ): Fig. $5 ;{ }^{13} \mathrm{C}$ NMR $(125 \mathrm{MHz}$, $\mathrm{D}_{2} \mathrm{O}$ ): Fig. 6 .

\section{Synthesis of HA-FA-NEG-OA-8 material}

HA-FA-NEG-OA-8 material was prepared by applying the above procedure to HA-FA-Pg-2F graft copolymer $(100 \mathrm{mg}$ ) and 3 (54 mg, $0.0768 \mathrm{mmol}$ ) at room temperature for $6 \mathrm{~h}$. HA-FANEG-OA-8 was obtained as a brown glassy solid $(80 \mathrm{mg}) .{ }^{1} \mathrm{H}$ NMR (500 MHz, $\left.\mathrm{D}_{2} \mathrm{O}\right)$ : see Fig. ESI- $1 . \dagger$

\section{SEC-MALS}

The molecular characterization was performed by a multi-angle laser light scattering (MALS) detector on line to a size exclusion chromatography (SEC or GPC) system. The SEC-MALS multidetector system consisted of an Alliance 2695 chromatograph from Waters (USA) with two on-line detectors: a MALS Dawn DSP-F photometer from Wyatt (USA) and a 410 differential refractometer (DRI) from Waters as concentration detector. In order to optimize sample solubility, regular elution, and SEC fractionation, an unusual solvent mixture [i.e. $0.2 \mathrm{M}$ aqueous $\mathrm{NaCl}$ solvent and dimethyl sulfoxide (DMSO) organic solvent $(50: 50 \mathrm{w} / \mathrm{w})]$ was used. The SEC experimental conditions were the following: two Polargel SEC columns ( $\mathrm{M}$ and L, $5 \mu \mathrm{m}$ of particle size) from Polymers Laboratories (UK), $35{ }^{\circ} \mathrm{C}$ of

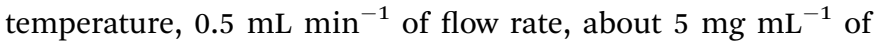
sample concentration.

The MALS calibration constant was calculated using toluene as standard by assuming a Rayleigh factor of $1.406 \times$ $10^{-5} \mathrm{~cm}^{-1}$. The MALS angular normalization was performed by measuring the scattering intensity of a concentrated solution of a pullulan polysaccharide with narrow molecular weight distribution (MWD, $M_{\mathrm{p}}=12 \mathrm{~kg} \mathrm{~mol}^{-1}, M_{\mathrm{w}} / M_{\mathrm{n}}<1.03, R_{\mathrm{g}}=\approx 2.1$ $\mathrm{nm}$ ) assumed to act as an isotropic scatterer. It is known that the on-line MALS detector measures, for each polymeric fraction eluted from the SEC columns, the molecular weight $(M)$ and when the angular dependence of the scattered light is experimentally measurable also the molecular size generally known as radius of gyration $\left(R_{\mathrm{g}}\right)$. The SEC-MALS system was described in detail elsewhere. ${ }^{26,27}$

The differential refractive index increment of the polymers with respect to the solvent mixture was measured off-line by a Chromatix KMX-16 differential refractometer.

\section{MALDI-TOF MS}

MALDI TOF MS (matrix assisted laser desorption/ionization time of flight mass spectrometry) mass spectra were carried out using a 4800 Proteomic Analyzer (Applied Biosystems) MALDI-TOF/TOF instrument equipped with a Nd:YAG laser at a wavelength of $355 \mathrm{~nm}$ with $<500$ ps pulse and $200 \mathrm{~Hz}$ firing rate, the acceleration voltage was set at $20 \mathrm{kV}$. The irradiance was maintained slightly above the threshold, to obtain a mass resolution of about 1000-2000 fwhm; isotopic resolution was observed throughout the entire mass range detected (from $\mathrm{m} / \mathrm{z}$ 1000 up to $m / z 6000$ ). External calibration was performed using an Applied Biosystems calibration mixture consisting of 

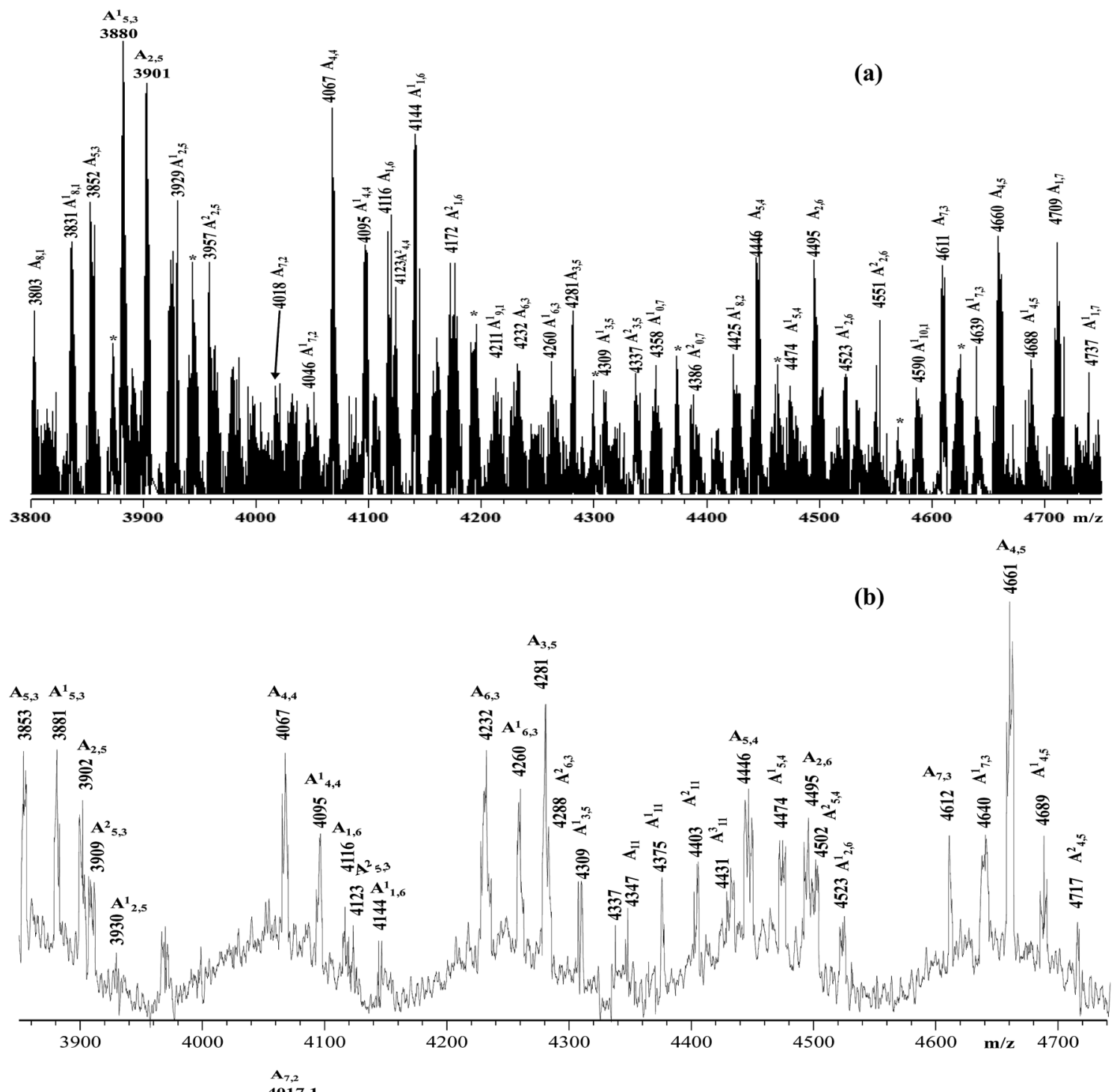

(c)

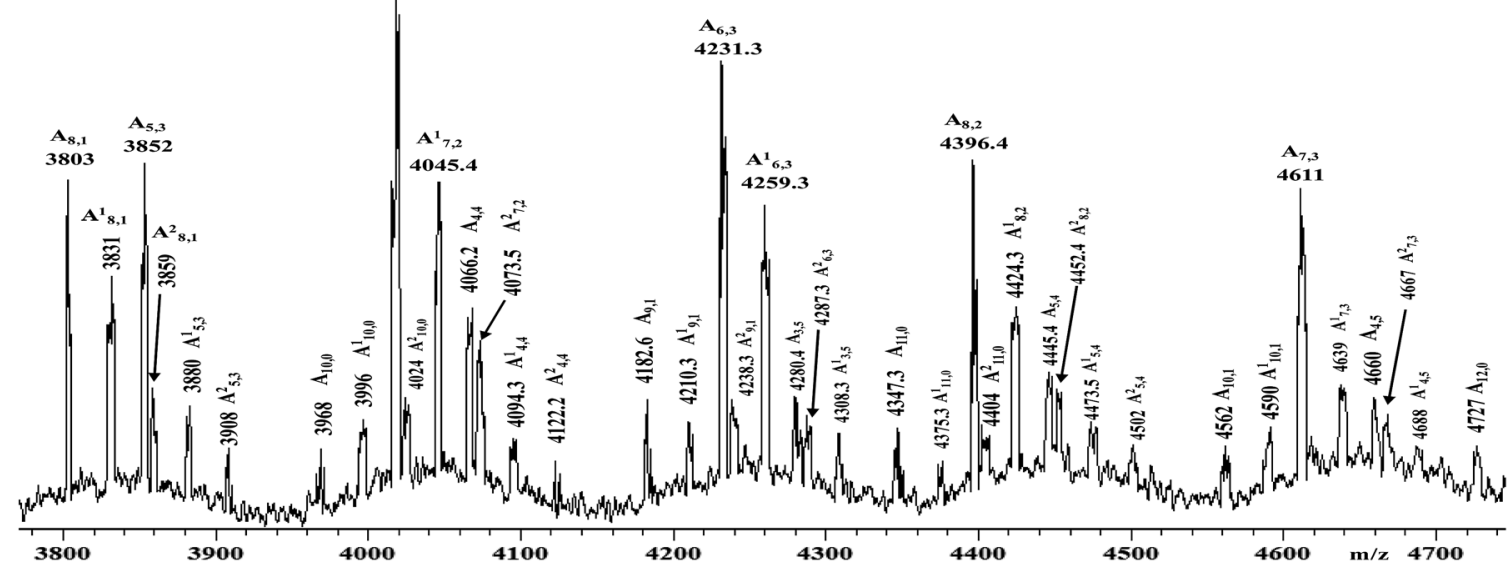

Fig. 2 Enlarged sections of the MALDI-TOF mass spectra (negative ions) of the samples: (a) HA-FA-Pg-2F; (b) HA-FA-Pg-3F; (c) HA-FA-Pg-4F.

polypeptides with different molecular weight values. Mass accuracy was about 150-200 ppm. All measurements were performed in negative ion mode; approximately 1500 laser shots were accumulated for each mass spectrum. The best spectra were recorded using 2,5-dihydroxybenzoic acid (2,5DHB) or trans-2-[3-(4-t-butyl-phenyl)-2-methyl-2-propenylidene] 


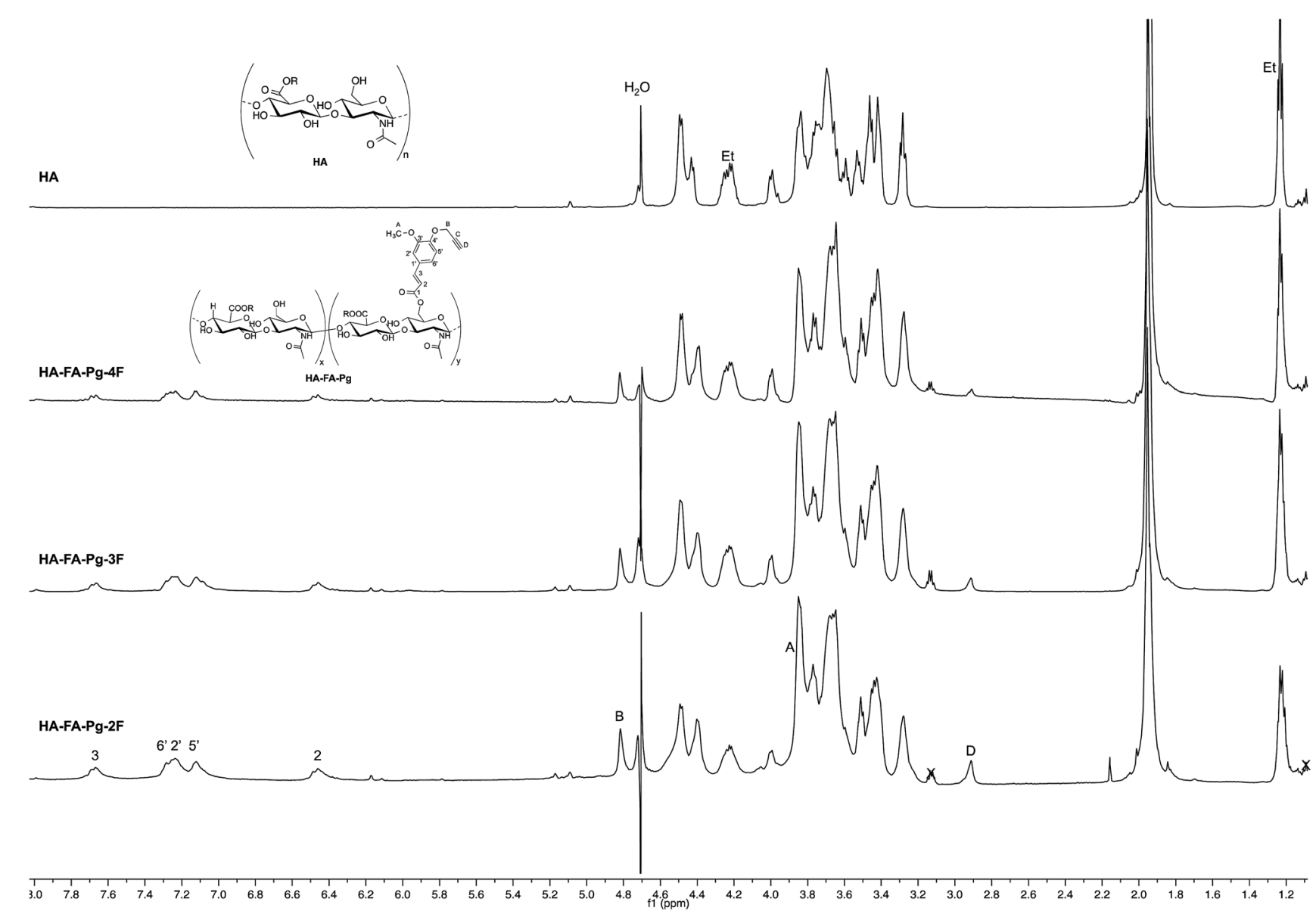

Fig. 3 Comparison of ${ }^{1} \mathrm{H}$ NMR spectra obtained with some selected $\mathrm{HA}-\mathrm{FA}-\mathrm{Pg}$ derivatives $\left(\mathrm{D}_{2} \mathrm{O}, 600 \mathrm{MHz}\right.$, with water suppression) with that obtained with starting $\mathrm{HA}$ sample. In the spectrum of $\mathrm{HA}$, Et labels indicate the signals of ethyl groups of the monomeric units showing $\mathrm{R}=\mathrm{C}_{2} \mathrm{H}_{5}$.

malononitrile (DCTB) as matrices $\left(0.1 \mathrm{M}\right.$ in $\mathrm{CH}_{3} \mathrm{CN} / \mathrm{CH}_{3} \mathrm{OH} 1 / 1$ $\mathrm{v} / \mathrm{v}$ ). Polymer samples were solubilized in the $\mathrm{CH}_{3} \mathrm{CN} / \mathrm{CH}_{3} \mathrm{OH}$ $(1: 1 \mathrm{v} / \mathrm{v})$ or $\mathrm{CHCl}_{3} / \mathrm{CH}_{3} \mathrm{OH}(2: 1 \mathrm{v} / \mathrm{v})$ solvent mixtures with a concentration of about $2 \mathrm{mg} \mathrm{mL}^{-1}$. Samples for MALDI analysis were prepared by the dried-droplet method, in which a mixture of matrix and sample $(0.3 \mu \mathrm{L})$ was deposited onto the target plate and dried at room temperature under inert atmosphere $\left(\mathrm{N}_{2}\right.$ flow).

\section{Dynamic light scattering (DLS) analysis and $\zeta$ potential measurements}

The mean diameter, width of distribution (polydispersity index, $\mathrm{PDI}$ ), and $\zeta$ potential of the nanoparticles were measured at $25^{\circ} \mathrm{C}$ using a Zetasizer NanoZS instrument fitted with a $532 \mathrm{~nm}$ laser at fixed scattering angle of $173^{\circ}$. The intensity-average hydrodynamic diameter (size in $\mathrm{nm}$ ) and PDI of the materials were measured in double distilled water. The $\zeta$ potential $(\mathrm{mV})$ was calculated from the electrophoretic mobility using the Smoluchowski relationship and assuming that $K \times a \gg 1$ (where $K$ and $a$ are the Debye-Hückel parameter and particle radius, respectively). Each experiment was performed in triplicate.

\section{Synthesis of liposomes}

Empty 1,2-dioleoyl-sn-glycerophosphocholine (DOPC)/1,2-dioleoyl-sn-glycerophosphoethanolamine (DOPE) liposomes were prepared at $1: 1 \mathrm{~mol}$ ratio with a total lipid concentration of 1.0 $\times 10^{-2}$ M. HA-FA-NEG-OA-8 material was loaded in zwitterionic liposomes. Liposomes were prepared in a round bottom vial by mixing the appropriate amounts of stock solutions, which were $3 \times 10^{-2} \mathrm{M}$ in chloroform for lipids, and $5 \mathrm{mg} \mathrm{mL}^{-1}$ in water for HA-FA-NEG-OA-8. A dry lipid film was obtained by evaporating the solvent under vacuum overnight. Rehydrating with Milli-Q grade $\mathrm{H}_{2} \mathrm{O}$ yielded multilamellar dispersion. Upon vortexing, multilamellar vesicles were obtained, which were then submitted to nine freeze/thaw cycles to improve the homogeneity of the size distribution in the final suspension. Liposomes were subsequently reduced in size and converted to unilamellar vesicles by extrusion through $100 \mathrm{~nm}$ polycarbonate membranes. Twenty-seven extrusions were performed with the LiposoFast apparatus (Avestin, Ottawa, Canada). All liposomes were stored at $4{ }^{\circ} \mathrm{C}$.

\section{Optical spectroscopy}

The UV-vis measurements were performed with a Perkin Elmer Lambda 900 spectrometer. PL spectra were obtained with a Nanolog spectrofluorimeter equipped with a Synapse QExtra CCD. The spectra were corrected for the instrument response. Photoluminescence (PL) quantum yield (QY) values of solutions were obtained by using quinine sulfate as the reference. PLQY 


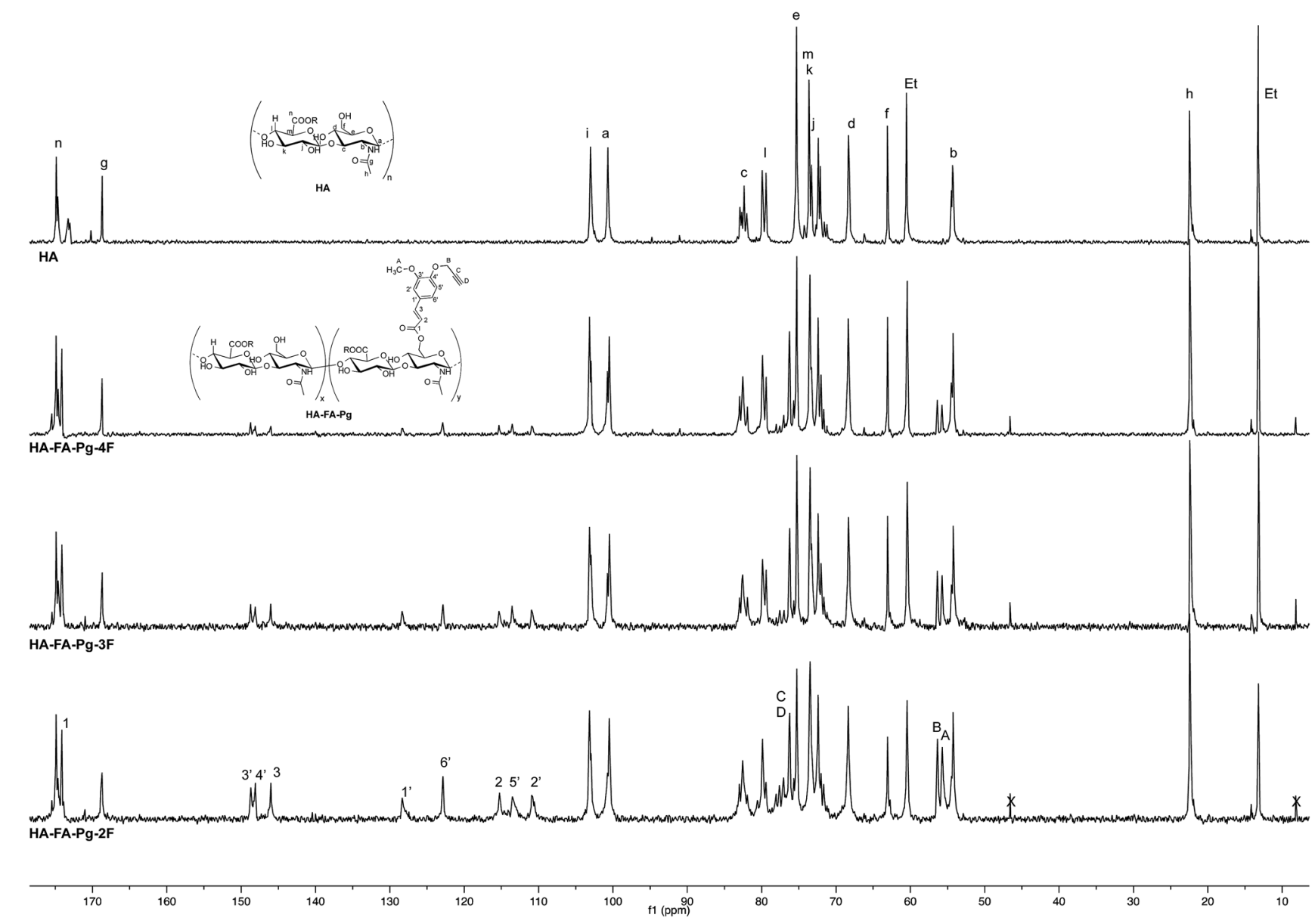

Fig. 4 Comparison of ${ }^{13} \mathrm{C}$ NMR spectra obtained with some selected $\mathrm{HA}-\mathrm{FA}-\mathrm{Pg}$ derivatives $\left(\mathrm{D}_{2} \mathrm{O}, 125 \mathrm{MHz}\right)$ with that obtained with starting $\mathrm{HA}$ sample. In the spectrum of $\mathrm{HA}$, Et labels indicate the signals of ethyl groups of the monomeric units showing $\mathrm{R}=\mathrm{C}_{2} \mathrm{H}_{5}$.

of solid powders were measured with a SPEX 270 M monochromator with a home-made integrating sphere according to the procedure reported elsewhere. ${ }^{28}$

\section{In vitro cell studies}

In order to evaluate the in vitro cytotoxicity of HA-FA-NEG-OA materials, the direct contact tests, proposed by ISO 10995-5, Biological evaluation of medical devices - Part 5: Tests for cytotoxicity: in vitro methods was used. This test is suitable for samples with various shapes, sizes or physical status (i.e. liquids or solids). The evaluation of in vitro acute toxicity does not depend on the final use for which the product is intended, and the document ISO 10995-5:2009 recommends many cell lines from American Type Collection. Among them, to test HA-FANEG-OA cytotoxicity, NIH3T3 mouse fibroblasts were chosen (see ESI $\dagger$ for the details). ${ }^{29}$ The direct contact test was also used for cytocompatibility evaluation by using Human Chondrocyte (HC). HC were propagated in chondrocyte growth medium and NIH3T3 in DMEM supplemented with 10\% fetal calf serum, 1\% L-glutamine-penicillin-streptomycin solution, and 1\% MEM non-essential amino acid solution, and incubated at $37{ }^{\circ} \mathrm{C}$ in a humidified atmosphere containing $5 \% \mathrm{CO}_{2}$. Once at confluence, the cells were washed with $0.1 \mathrm{M}$ PBS, separated with
trypsin-EDTA solution and centrifuged at $1000 \mathrm{rpm}$ for 5 minutes. The pellet was re-suspended in complete medium (dilution $1: 15$ ).

PANC-1 (human pancreas adenocarcinoma) cells for confocal microscopy studies were obtained from American Type Collection and cultured as described above.

\section{Fluorescence imaging}

The internalization of HA-FA-NEG-OA materials in NIH3T3 cells was analyzed by fluorescence microscopy, and in PANC-1 cells by confocal microscopy.

Fluorescence microscopy. Cover slips were deposited on the wells of a 6 -well plate. $1.5 \times 10^{5}$ cells were seeded in each well. Cells were incubated with $7 \times 10^{-2} \mathrm{mg} \mathrm{mL}^{-1}$ concentration of HA-FA-NEG-OA-4 for $15 \mathrm{~min}$ at $37^{\circ} \mathrm{C}$. Then, cells were washed with PBS, fixed with $4 \%$ paraformaldehyde for $10 \mathrm{~min}$ at room temperature and visualized using an inverted fluorescent microscope (Axiovert 200, Zeiss, Germany).

Confocal microscopy. Cover glass slides were deposited on the bottom of the wells of a 24 -well plate. $6 \times 10^{4}$ PANC- 1 cells were seeded in each well. After $24 \mathrm{~h}$, cells were incubated with 3 $\times 10^{-2} \mathrm{mg} \mathrm{mL}^{-1}$ of HA-FA-NEG-OA materials for $30 \mathrm{~min}$ at $37^{\circ} \mathrm{C}$. The cells were then incubated with medium for 1,2 or $4 \mathrm{~h}$ 


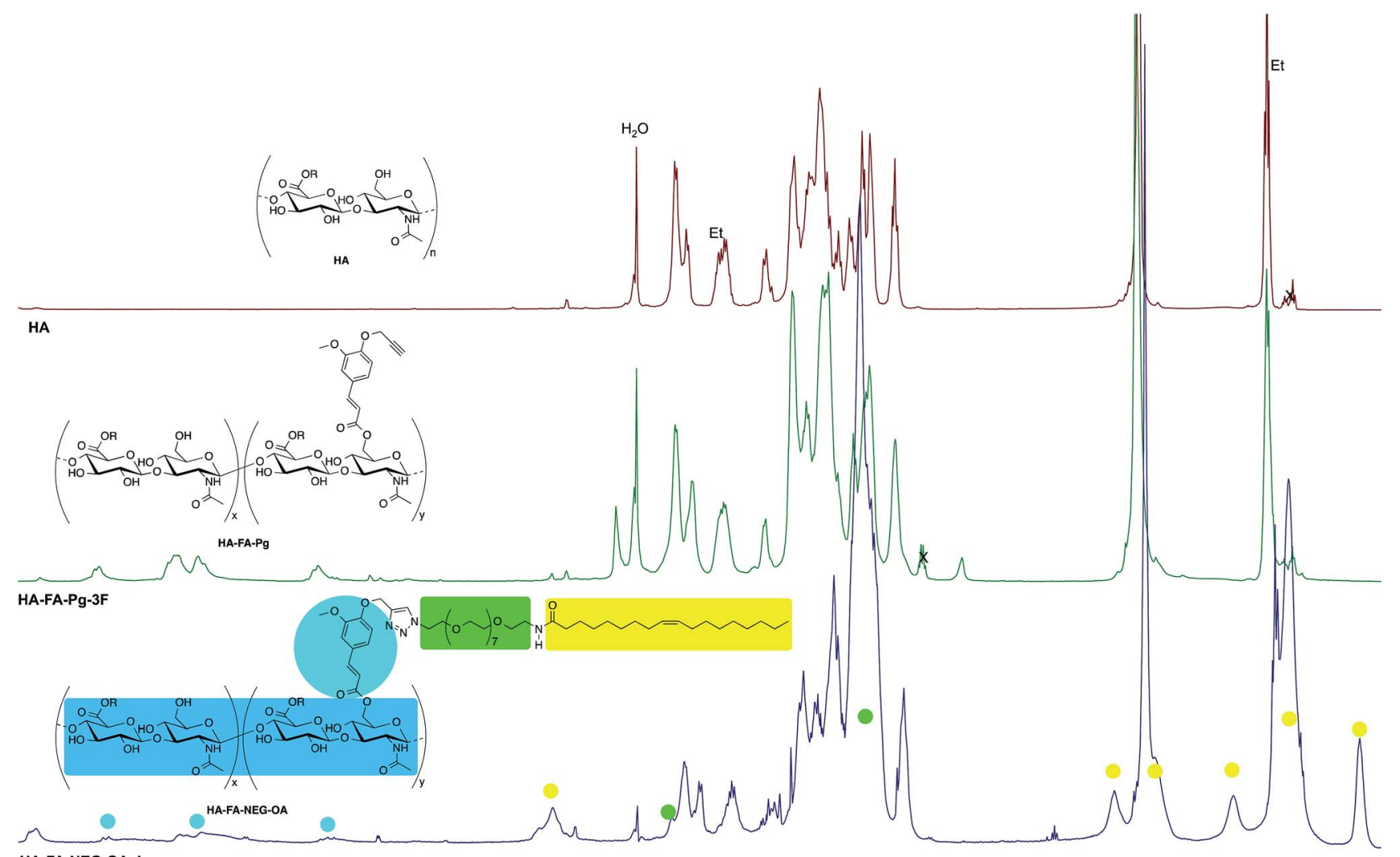

HA-FA-NEG-OA-4

Fig. 5 Comparison of ${ }^{1} \mathrm{H}$ NMR spectrum obtained with $\mathrm{HA}-\mathrm{FA}-\mathrm{NEG}-\mathrm{OA}-4$ derivative $\left(\mathrm{D}_{2} \mathrm{O}\right.$, with water suppression) with those obtained with starting $\mathrm{HA}-\mathrm{FA}-\mathrm{Pg}-3 \mathrm{~F}$ and $\mathrm{HA}$ samples. In the spectrum of $\mathrm{HA}$, Et labels indicate the signals of ethyl groups of the monomeric units showing $\mathrm{R}=$ $\mathrm{C}_{2} \mathrm{H}_{5}$.

at $37^{\circ} \mathrm{C}$. Then cells were fixed with $4 \%$ paraformaldehyde for $10 \mathrm{~min}$ at room temperature and stained with Lectin-FITC $(1: 3000$ in PBS-BSA 1\%) for $10 \mathrm{~min}$ at room temperature in the dark. After each step were performed two washes with PBS. Glasses were then mounted with Mowiol and visualized using a confocal microscope (SP5, Leica, Germany).

\section{Results and discussion}

\section{Synthesis of HA-FA-Pg and HA-FA-NEG-OA derivatives}

The hyaluronan derivatives HA-FA-Pg were prepared by reaction of low molecular weight $\mathbf{H A}$ with imidazolide $\mathbf{1}^{24}$ as reported in Scheme 1.

The functionalization reaction was carried out in formamide as the solvent and in the presence of triethylamine (TEA) as the base to afford the desired graft copolymer HA-FA-Pg. ${ }^{24}$ The stoichiometric ratio between $\mathbf{1}$ and $\mathbf{H A}$ (i.e. 1/HA ratio, Table 1) was varied from 50 to $12.5 \%$ with a number of replicates in $\mathbf{1} / \mathbf{H A}$ ratios of $25 \%$ and $50 \%$ in the aim of evaluating the reproducibility of the molar grafting degree (GD) observed. The isolation of the copolymer from the reaction mixture was performed by precipitation with acetone to obtain samples as white solids.
The analysis of the data reported in Table 1 demonstrate that GD value can be regulated in the range $10-35 \%$ by using the correct 1/HA stoichiometric ratio, with high and quite reproducible conversion values (i.e. $60-80 \%$ ).

Some selected HA-FA-Pg samples were then used in the CuAAC coupling with alpha-azido-omega-oleic amide nona(ethylene glycol) (azido-NEG-OA, 3) to obtain HA derivatives (i.e. HA-FA-NEG-OA) as depicted in Scheme 2.

The copper(I) catalyst was generated in situ with $\mathrm{CuSO}_{4} /$ sodium ascorbate in order to perform the CuAAC coupling under very mild conditions. The coupling reaction was carried out by using two HA-FA-Pg samples showing different grafting degrees (i.e. about $20 \%$ corresponding to four propargyl groups per HA macromolecule in HA-FA-Pg$3 \mathrm{~F}$, and about $35 \%$ corresponding to eight propargyl groups per HA macromolecule in HA-FA-Pg-2F) in the aim of obtaining HA-FA-NEG-OA derivatives bearing different densities of $\mathbf{O A}$ side chains pending from the HA backbone (i.e. about four in HA-FA-NEG-OA-4, and about eight in HA-FA-NEG-OA-8).

Finally, compound FA-NEG-OA and its methyl ester precursor 5 were synthesized as described in Scheme 3 in order to be used as model compounds. 


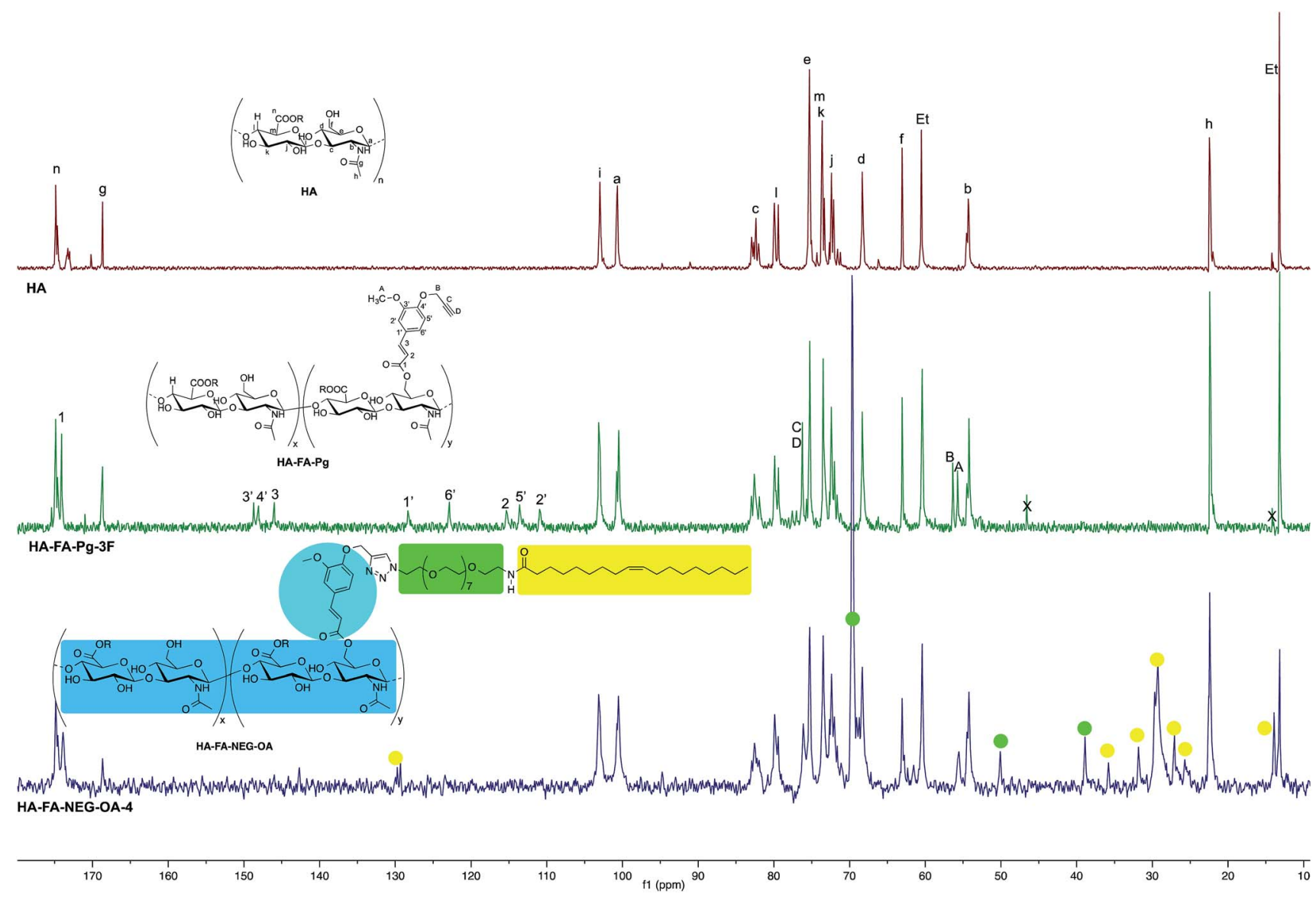

Fig. 6 Comparison of ${ }^{13} \mathrm{C}$ NMR spectrum obtained with HA-FA-NEG-OA-4 derivative $\left(125 \mathrm{MHz}, \mathrm{D}_{2} \mathrm{O}\right)$ with those obtained with starting HA$\mathrm{FA}-\mathrm{Pg}-3 \mathrm{~F}$ and $\mathrm{HA}$ samples. In the spectrum of $\mathrm{HA}$, Et labels indicate the signals of ethyl groups of the monomeric units showing $\mathrm{R}=\mathrm{C}_{2} \mathrm{H}_{5}$.

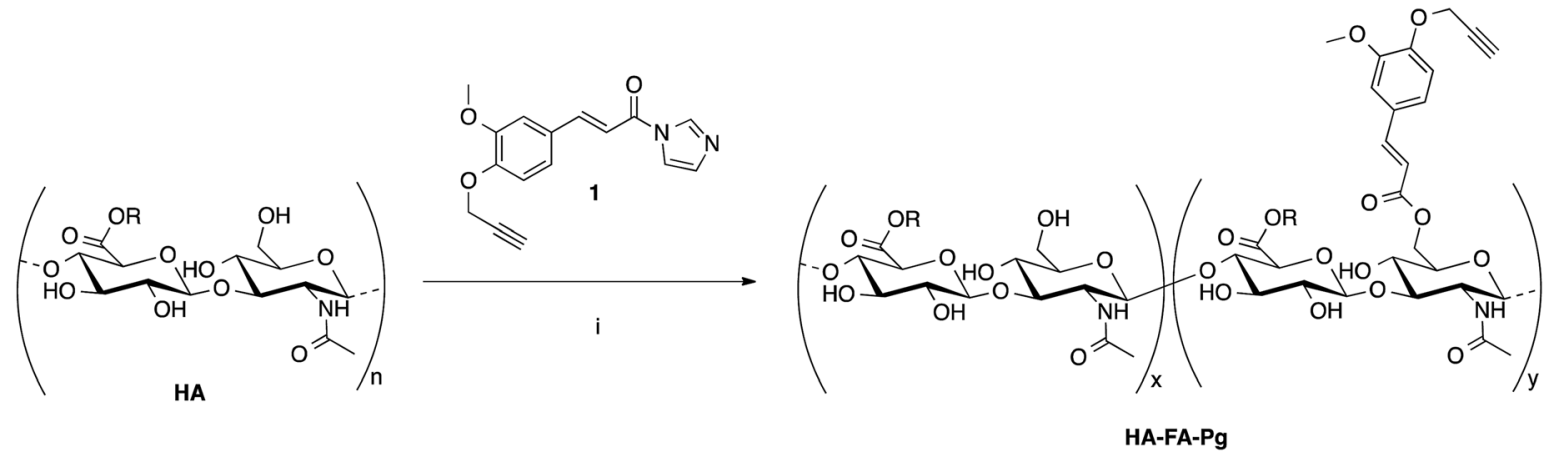

Scheme 1 Preparation of the HA-FA-Pg derivatives. Reagents: (i) $\mathrm{HCONH}_{2}$, TEA. Substituents: $\mathrm{R}=\mathrm{H}$ or $\mathrm{C}_{2} \mathrm{H}_{5}$.

\section{Molecular characterization of HA-FA-Pg derivatives}

The molecular characterization of HA-FA-Pg graft copolymers and of the starting HA was performed by means of a multi-angle laser light scattering (MALS) absolute detector on-line to a size exclusion chromatography (SEC) system by using a suitable solvent mixture [i.e. $0.2 \mathrm{M} \mathrm{NaCl-DMSO}(80: 20)]$ as the mobile phase. The most important SEC-MALS results obtained [i.e. the molecular weight of the peak of the chromatogram $\left(M_{\mathrm{p}}\right)$, the weight-average of the molecular weight $\left(M_{\mathrm{w}}\right)$, and the polydispersity index $M_{\mathrm{w}} / M_{\mathrm{n}}$ where $M_{\mathrm{n}}$ denotes the numericaverage of the molecular weight] are summarized in Table 2. The table also reports the recovered mass that is the fraction of polymeric sample eluting from the SEC columns, the $\mathrm{d} n / \mathrm{d} c$, and the grafting degree values.

In consideration of the complex copolymer structure and the unusual SEC solvent mixture, the recovered mass values of the samples were relative high and substantially adequate for estimating the whole MWD of the copolymers, particularly after the 


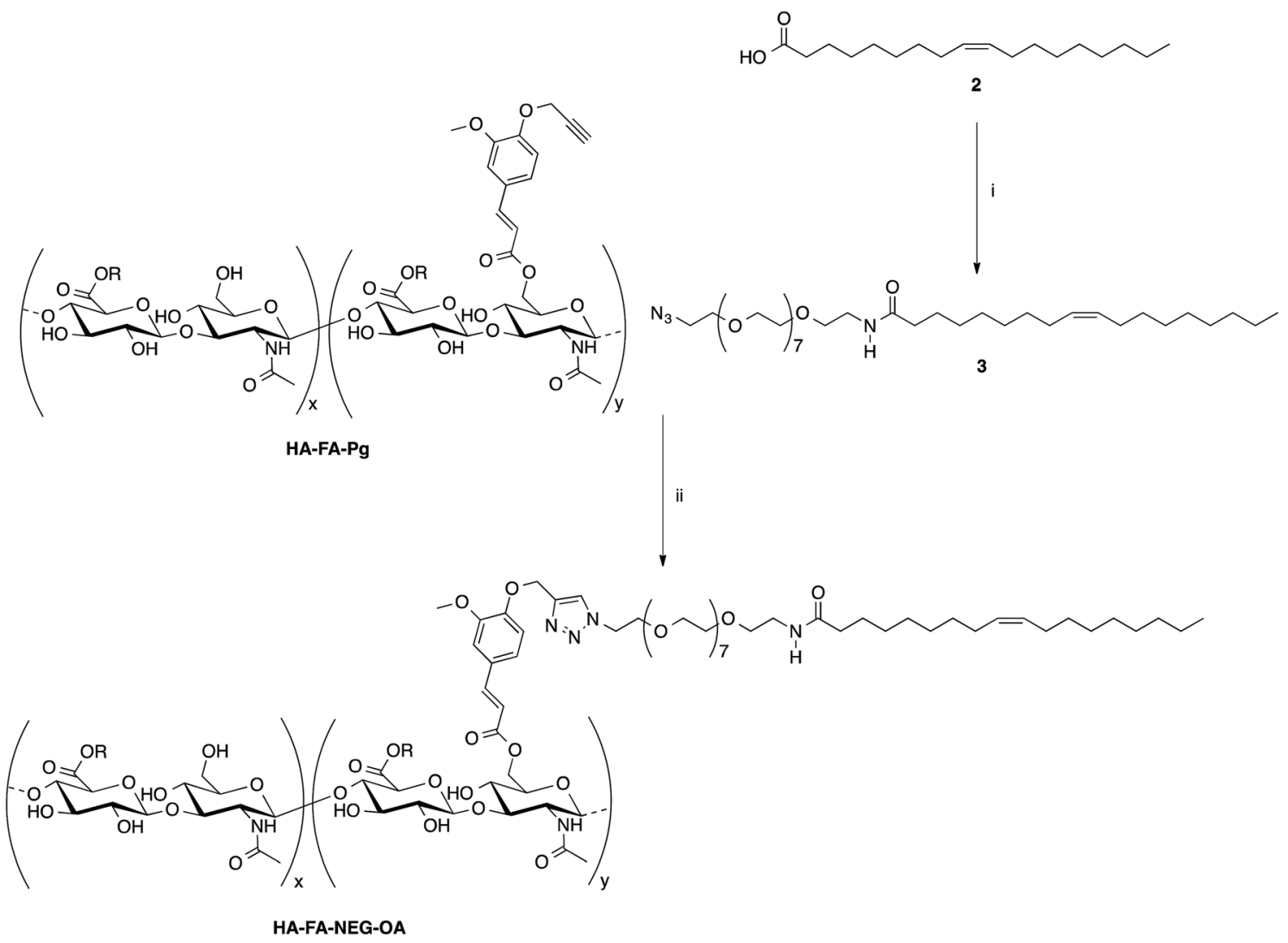

Scheme 2 Click chemistry functionalization of $\mathrm{HA}-\mathrm{FA}-\mathrm{Pg}$ with azido-NEG-OA leading to HA-FA-NEG-OA. Reagents: (i) CDI, THF, $\mathrm{N}_{3} \mathrm{CH} \mathrm{H}_{2}$ $\mathrm{CH}_{2}\left(\mathrm{OCH}_{2} \mathrm{CH}_{2}\right)_{8} \mathrm{NH}_{2}$; (ii) $\mathrm{CuSO}_{4}$, sodium ascorbate, tert-BuOH, $\mathrm{H}_{2} \mathrm{O}$. Substituents: $\mathrm{R}=\mathrm{H}$ or $\mathrm{C}_{2} \mathrm{H}_{5}$.

derivatization reactions. Consequently, the SEC-MALS results in Table 2 provide interesting and very useful information for the native HA and the HA-FA-Pg derivatives.

The molecular weight of the starting HA sample was relatively low for a HA polysaccharide $\left(M_{\mathrm{w}}\right.$ average about $8.7 \mathrm{~kg}$ $\left.\mathrm{mol}^{-1}\right)$ and the MWD was substantially broad $\left(M_{\mathrm{w}} / M_{\mathrm{n}}\right.$ about 1.5). On the whole, in consequence of grafting the MWD of HAFA-Pg derivatives change only a little (i.e. the $M_{\mathrm{w}}$ average decrease from $8.7 \mathrm{~kg} \mathrm{~mol}^{-1}$ of the starting $\mathbf{H A}$ to $6.3 \mathrm{~kg} \mathrm{~mol}^{-1}$ of the HA-FA-Pg-2F derivative showing 35\% of grafting). During derivatization reactions two molecular weight tendencies are simultaneously working in opposite ways: (1) a molecular weight decrease produced by an eventual degradation; (2) a molecular weight increase produced by the grafting. The data reported in Table 2 demonstrated that degradation of HA starting biopolymer is relatively low also for high level of grafting. Furthermore, the $\mathrm{d} n / \mathrm{d} c$ values appeared to be quite well related with the grafting degree and this result substantially confirmed the estimation of the grafting.

Functionalized HA-FA-Pg samples (i.e. HA-FA-Pg-2F, HAFA-Pg-3F, and HA-FA-Pg-4F) were characterized by MALDI-TOF MS in negative-ion mode using DHB or DCTB as the matrix. The best mass resolved mass spectra of the HA-FA-Pg-3F and HAFA-Pg-4F samples were recorded using the DHB as the matrix, and the DCTB one for the HA-FA-Pg-2F derivative. As previously reported, ${ }^{24}$ the mass spectrum of starting HA showed, in the mass range $m / z$ 2500-10 $000 \mathrm{Da}$, a series of clusters corresponding to the even-numbered HA oligosaccharides (7- to 23mers, species $\mathrm{A}_{n}$ ). On the other hand, complex mass spectra were obtained in the case of the hyaluronan graft copolymers HA-FA-Pg-2F, HA-FA-Pg-3F, and HA-FA-Pg-4F, which shows a series of cluster of peaks in the mass range $\mathrm{m} / \mathrm{z} 3500-10000$. Enlarged sections of these mass spectra are reported in Fig. 2 together with the structural assignment. The analysis of the spectra suggested that the most intense peaks belong to the expected graft copolymer chains (species $\mathrm{A}_{x, y}$ ). In particular, most of the copolymer chains present along the backbone: 6-8 ferulate residues in the case of HA-FA-Pg-2F, 4-5 ferulate residues in the case of HA-FA-Pg-3F, 2-3 ferulate residues in the case of HA-FA-Pg-4F. The corresponding copolymer chains containing ethyl ester groups (species $\mathrm{A}_{x, y}^{\prime}$ ) were also revealed in accord to the mass spectrum of the initial HA sample. The mass spectra revealed also the presence of unfunctionalized oligosaccharides, in particular in the case of the HA-FA-Pg-3F and the HA-FA-Pg-4F samples (Fig. 2). Thus, the MALDI mass spectra confirmed the formation of the reactive functionalized oligosaccharides.

\section{Structure of HA-FA-Pg and HA-FA-NEG-OA derivatives}

The structure of HA-FA-Pg and HA-FA-NEG-OA derivatives was investigated by ${ }^{1} \mathrm{H}$ and ${ }^{13} \mathrm{C}$ NMR spectroscopic studies using $\mathrm{D}_{2} \mathrm{O}$ as the solvent. A comparative analysis was performed on 


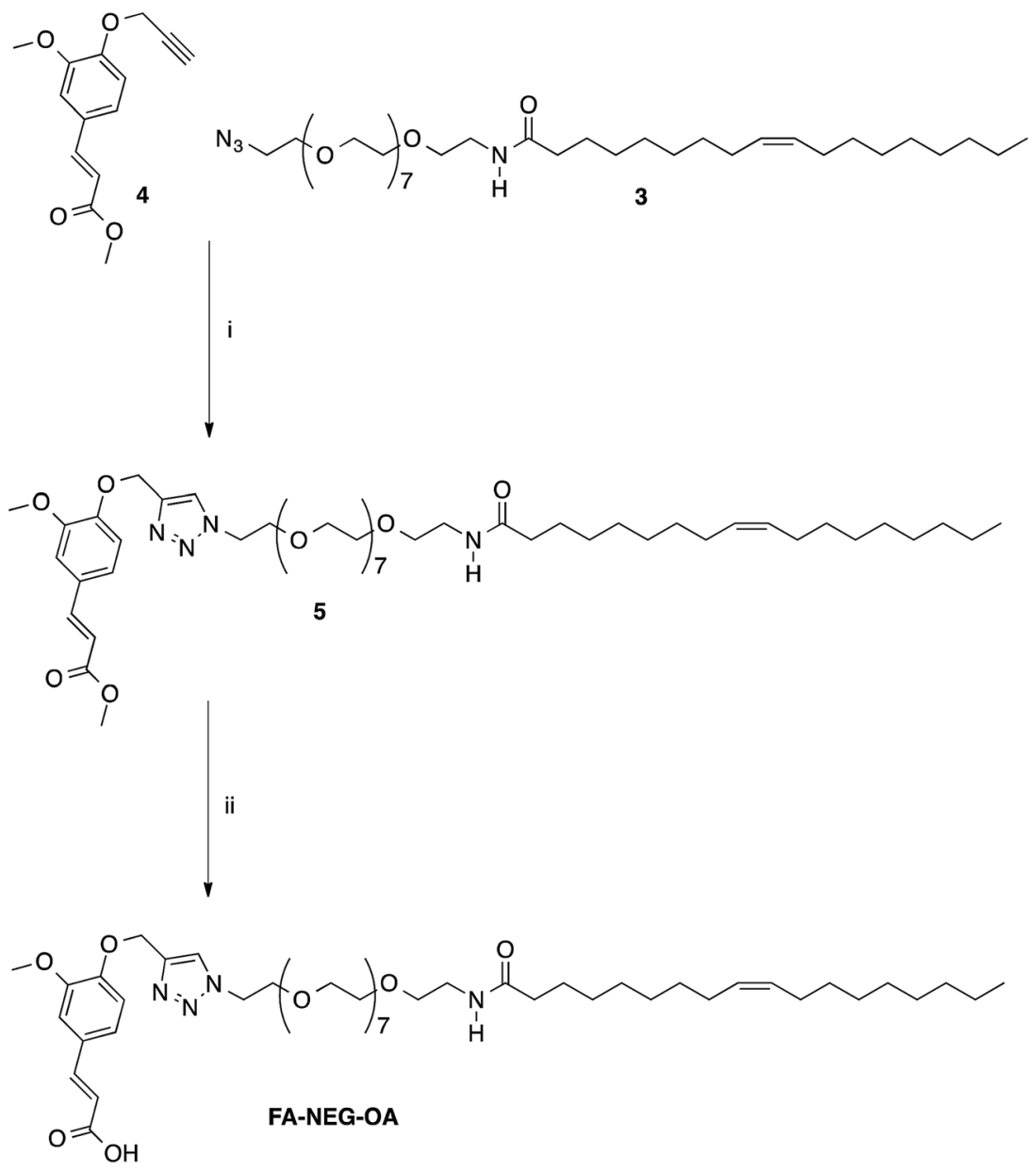

Scheme 3 Synthesis of model compounds 5 and FA-NEG-OA. Reagents: (i) $\mathrm{CuSO}_{4}$, sodium ascorbate, tert- $\mathrm{BuOH}, \mathrm{H}_{2} \mathrm{O}$; (ii) $\mathrm{NaOH}$, $\mathrm{H}_{2} \mathrm{O}$, $\mathrm{C}_{2} \mathrm{H}_{5} \mathrm{OH}$.

Table 2 Macromolecular features of starting HA and HA-FA-Pg graft copolymers

\begin{tabular}{|c|c|c|c|c|c|c|}
\hline Sample & $\mathrm{d} n / \mathrm{d} c\left(\mathrm{~mL} \mathrm{~g}^{-1}\right)$ & $M_{\mathrm{p}}\left(\mathrm{kg} \mathrm{mol}{ }^{-1}\right)$ & $M_{\mathrm{w}}\left(\mathrm{kg} \mathrm{mol}{ }^{-1}\right)$ & $M_{\mathrm{w}} / M_{\mathrm{n}}$ & Rec. mas. ${ }^{a}(\%)$ & Grafting $^{b}(\%)$ \\
\hline HA & 0.116 & 8.5 & 8.7 & 1.53 & 70 & 0 \\
\hline HA-FA-Pg-1F & 0.125 & 8.4 & 7.3 & 1.40 & 61 & 33 \\
\hline HA-FA-Pg-3F & 0.122 & 6.8 & 6.5 & 1.45 & 56 & 20 \\
\hline HA-FA-Pg- 4 F & 0.120 & 7.0 & 6.7 & 1.22 & 66 & 10 \\
\hline
\end{tabular}

${ }^{a}$ Recovered mass: the fraction of the polymeric sample eluting from the SEC columns. ${ }^{b}$ A rough estimate of the grafting degree was made by ${ }^{1} \mathrm{H}$ NMR spectroscopy after hydrolysis with $\mathrm{NaOD}$ in $\mathrm{D}_{2} \mathrm{O}$ as described in ref. 22.

the ${ }^{1} \mathrm{H}$ NMR spectra obtained with HA-FA-Pg samples prepared by using different stoichiometric ratio between 1 and HA (i.e. 1/ HA ratio from 50 to $12.5 \%$, see Table 1 ). In particular, two HAFA-Pg samples were selected on the basis of their grafting degree spanning from $35 \%$ (HA-FA-Pg-2F) to $10 \%$ (HA-FA-Pg$2 \mathrm{~F}$ ) in comparison with the previously published sample HAFA-Pg-3F showing an intermediate grafting degree (i.e. around 20\%). ${ }^{24}$ The ${ }^{1} \mathrm{H}$ NMR experiments (Fig. 3) confirmed the successful coupling between $\mathbf{H A}$ and $\mathbf{1}$ in our reaction conditions.
The ${ }^{1} \mathrm{H}$ NMR spectra indeed showed, beside the typical profile of HA in the up-field region, signals attributed to the propargylated FA residues in the down-field region of the recorded spectra. Moreover, the relative intensity of the signals in the aromatic region increased from HA-FA-Pg- $4 \mathrm{~F}$ (grafting degree around $10 \%$ ) to HA-FA-Pg-3F (grafting degree around $20 \%$ ) and HA-FA-Pg-2F (grafting degree around 35\%) with the increase of the grafting degree. This observation along with the broadness of the typical signal pattern in the aromatic region supported further the occurrence of the functionalization. 
The more resolved spectral lines allowed a more detailed comparison of the ${ }^{13} \mathrm{C}$ NMR spectra (Fig. 4) to be done.

The assignment of the ${ }^{13} \mathrm{C}$ NMR spectra of HA and HA-FA-Pg samples was performed as previously described.$^{24}$ In agreement with that observed in the analysis of ${ }^{1} \mathrm{H}$ NMR spectra, the relative intensity of the signals attributed to propargylated FA residues increased with the increase of the grafting degree, which appeared to play a negligible role in modulating the shoulder of the signals attributed to anomeric $\mathrm{C}$ - a carbon atom of HA.

In order to evaluate the occurrence of the click chemistry coupling between the selected HA-FA-Pg samples (i.e. HA-FAPg-2F and HA-FA-Pg-3F) leading HA-FA-NEG-OA derivatives (i.e. HA-FA-NEG-OA-8 and HA-FA-NEG-OA-4, respectively), the ${ }^{1} \mathrm{H}$ and ${ }^{13} \mathrm{C}$ NMR spectra of HA-FA-NEG-OA-4 were analyzed in comparison with those of its synthetic precursor and HA-FA-Pg-3F (Fig. 5 and 6) and of the model compound FA-NEG-OA, which was extensively characterized by means 1D and 2D NMR techniques. In this way, we were able to assign all the signals of the complex spectra obtained with HA-FA-NEGOA materials (see ESI $\dagger$ ).

In fact, the ${ }^{1} \mathrm{H}$ NMR spectrum of the HA-FA-NEG-OA-4 material (Fig. 5) appeared to be composed by a multitude of signals deriving from the integration of discrete signal patterns relevant to the different components of the material.

Interestingly, the disappearance of the peak at $2.91 \mathrm{ppm}$ attributed to acetylene proton of HA-FA-Pg-3F and the appearance of a new peak at 8.02 in the spectrum of HA-FANEG-OA-4 material supported the transformation of the alkyne moiety into the triazole one as it usually occurs in CuAAC coupling reaction. Therefore, this observation was considered to be a strong evidence of the structure of FA-NEG-OA-4 material. Furthermore, a significant line broadening was observed in the signals attributed to the aromatic and acrylic protons of FA moiety.

Similarly, the ${ }^{13} \mathrm{C}$ NMR spectrum of the HA-FA-NEG-OA-4 material (Fig. 6) resulted by the combination of the signal patterns relevant to the different components of the material. Among them, the signals attributed to the low molecular weight HA ribbons and to NEG side chains dominated the spectrum, with the one attributed oleic acid moiety playing a relatively minor role, and the ones belonging to the aromatic carbon atoms of ferulate component being lost in the baseline modulations. In agreement with previous observations, ${ }^{24}$ we assumed that owing to its role of linker between the two macromolecular components (i.e. HA and OEG-OA), the FA moiety suffered from

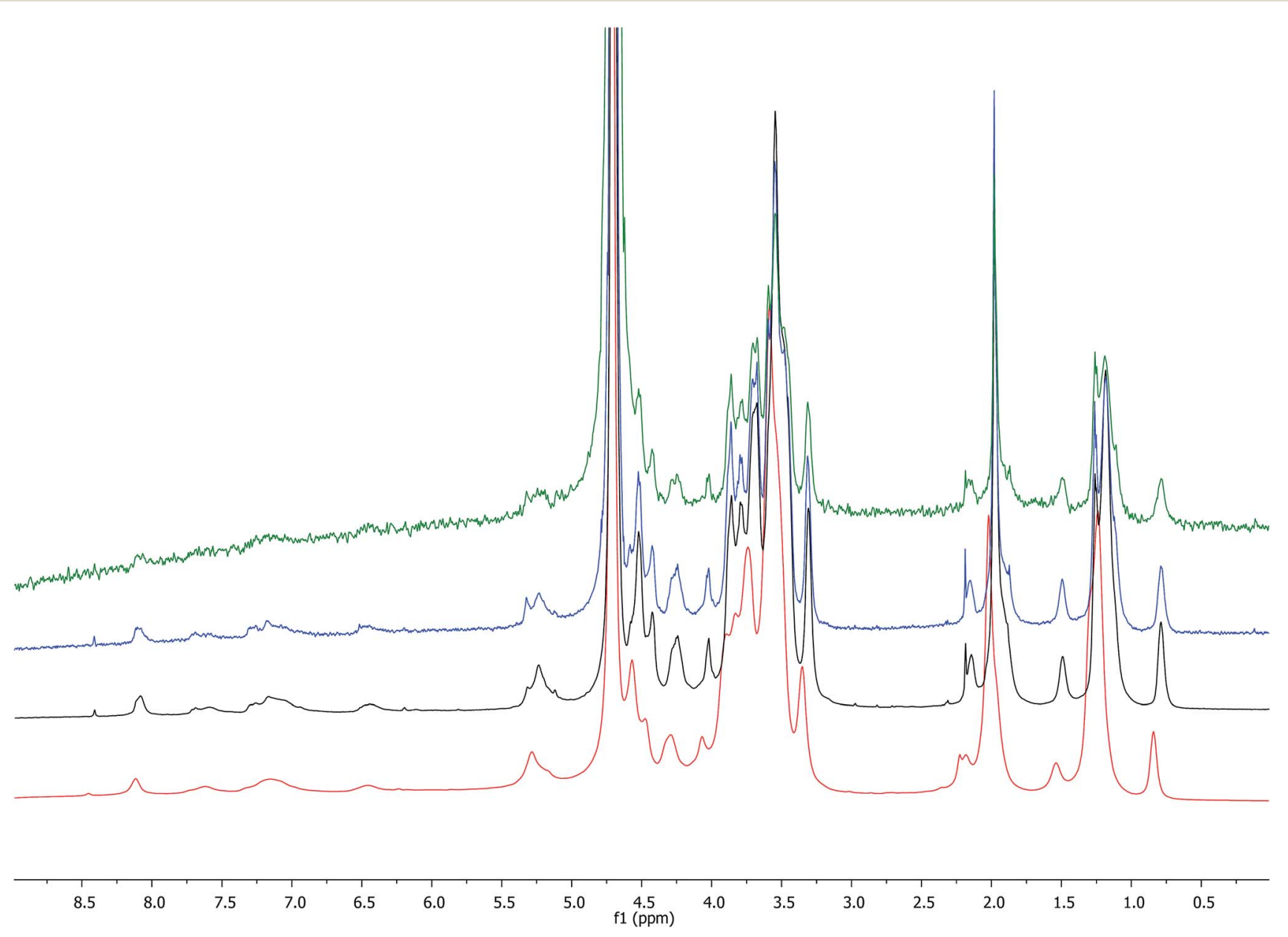

Fig. 7 Comparison of ${ }^{1} \mathrm{H}$ NMR spectra $\left(600 \mathrm{MHz}\right.$ ) obtained with increasing concentrations of HA-FA-NEG-OA-4 derivative in $\mathrm{D}_{2} \mathrm{O}$ (red: $25 \mathrm{mg} \mathrm{mL}^{-1}$, black: $2.5 \mathrm{mg} \mathrm{mL}^{-1}$, blue: $0.25 \mathrm{mg} \mathrm{mL}^{-1}$, green: $0.025 \mathrm{mg} \mathrm{mL}^{-1}$ ). 
a reduced mobility, which could produce a pronounced line broadening as already observed in the ${ }^{1} \mathrm{H}$ NMR spectrum in Fig. 5 .

Nevertheless, the persistence of the signal attributed to the ferulate methoxy C-A (at about $55.6 \mathrm{ppm}$ ) and the apparent shift of both the one attributed to the ferulate propargyl C-B (at about $56.4 \mathrm{ppm}$ ) and the one attributed to the ferulate propargyl carbons C-D and C-C (at about $76 \mathrm{ppm}$ ) were assumed to confirm the occurrence of the CuAAC coupling reaction.

The comparison of the ${ }^{1} \mathrm{H}$ NMR spectra obtained with increasing concentrations of HA-FA-NEG-OA-4 in $\mathrm{D}_{2} \mathrm{O}$ (Fig. 7) showed a gradual broadening, which suggested a tendency towards aggregation of this material.

This result led us to assume the formation of colloidal water dispersions with HA-FA-NEG-OA-4 in the form of micelles with a hydrophobic core constituted by the oleic residues. The high field (900 MHz) NOESY spectrum (Fig. 8) performed on the water dispersions of the material showed the presence of strong dipolar interactions among different portions of the macromolecules, which should be far in extended conformations, but were evidently near from each other in the 3D space.
This result suggested the existence of a strong entanglement of the side chain components (both the lipophilic oleic one and the amphiphilic NEG one) in the core of the micelles, which exposed on their surface the hydrophilic side of the hyaluronan ribbon as suggested by the apparent lack of dipolar interactions of the acetamide methyl group (sharp singlet at $1.92 \mathrm{ppm}$ ) with the side chain components.

The relative sign of cross-peaks in a NOESY spectrum depends on the rotational correlation time, i.e. if the rotational correlation time of the molecules is long, the diagonal and cross-peaks have same signs. ${ }^{30}$ This result allowed assertion that the HA-FA-NEG-OA-4 experienced a slower motion in solutions confirming the aggregation processes.

\section{Dynamic light scattering (DLS) characterization of HA-FA- NEG-OA derivatives in water}

The aim of these experiments was to study the behavior of HAFA-NEG-OA materials in water solution in relation to the variation of size with respect to concentration. The presence of oleic acid could lead to the formation of spherical or more probably

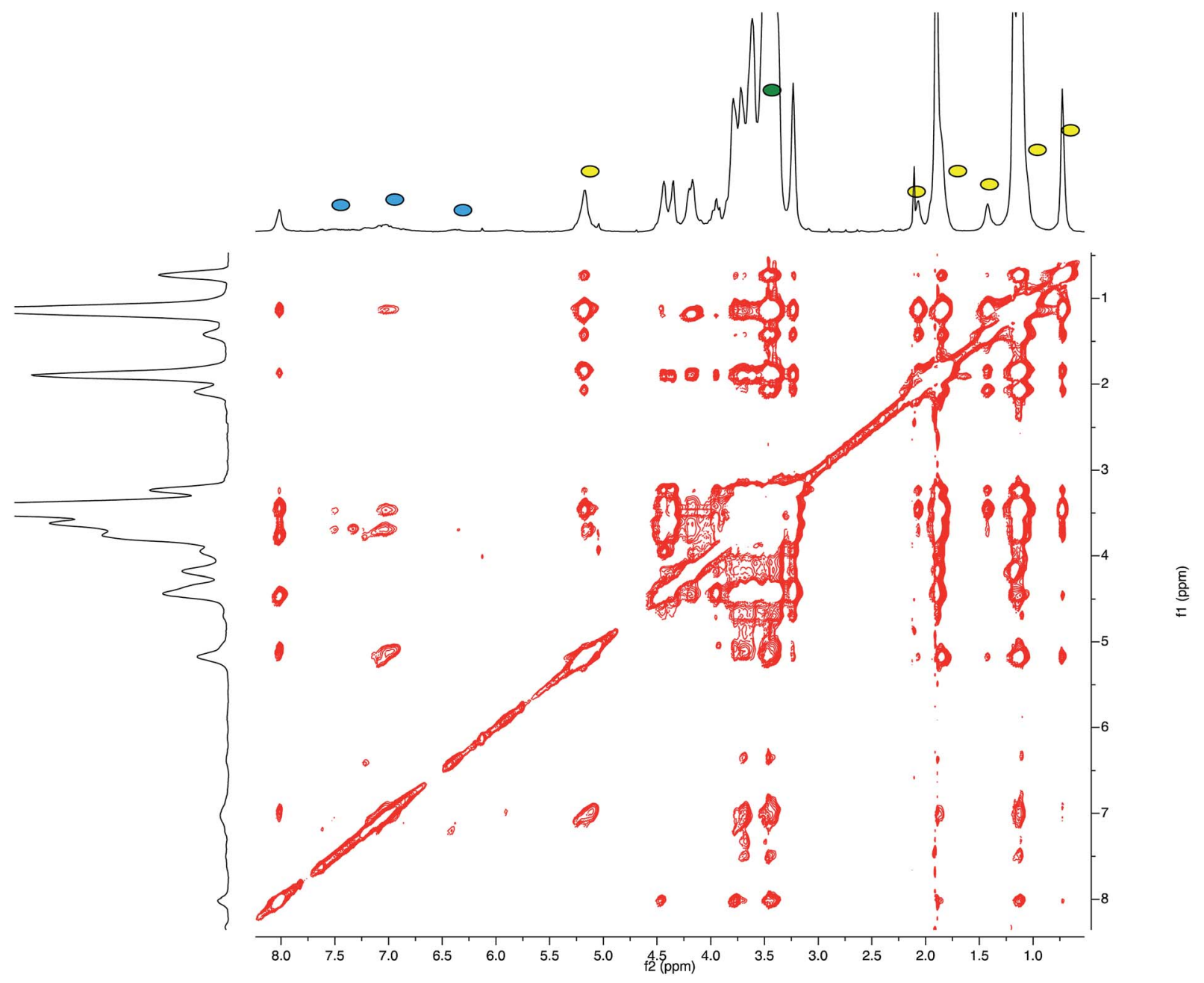

Fig. 8 High field (900 MHz TCl cryo) NOESY spectrum (mixing time $200 \mathrm{~ms}$ ) performed on the water (i.e. $\mathrm{D}_{2} \mathrm{O}$, with water suppression) dispersions of HA-FA-NEG-OA-4 material. 


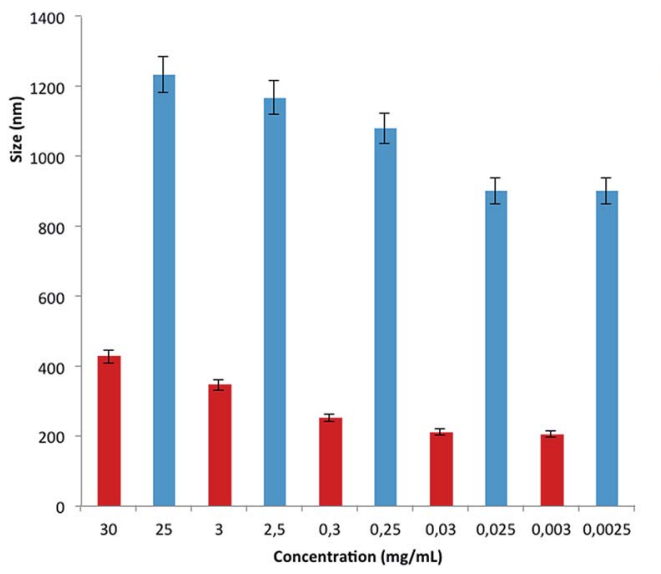

Fig. 9 Mean particle size of HA-FA-NEG-OA material versus concentration of the water dispersions.

worm-like micelles in solution. Fig. 9 reports the DLS data for HA-FA-NEG-OA materials.

At high concentration the HA-FA-NEG-OA-4 forms very large aggregates, whose polydispersity index (PDI) was reasonable even if quite high. In this case, the distribution curve obtained from Laplace reversal for such large diameter values may contain artifacts. The aggregate size analysis was, furthermore, limited to the restrictions of DLS, where the aggregate must remain small enough for Brownian forces to dominate gravitational force, preventing sedimentation, which for polymeric particles is generally considered to be $1000 \mathrm{~nm}^{31}$ The high polydispersity indexes revealed that the systems were not perfectly monodispersed. The size decrease with concentration and this experimental data highlight that the macromolecule aggregate structure was similar to spherical or a worm-like micelles.

HA-FA-NEG-OA-8 at the maximum tested concentration of $30 \mathrm{mg} \mathrm{mL} \mathrm{m}^{-1}$ had two main distributions, whose data were not, however, very reproducible. This is typical of polymeric solutions in which macromolecules do not have a well-defined form. HA-FA-NEG-OA-8 produced smaller aggregate than HA-FANEG-OA-4. However, these experimental data suggest that the both HA-FA-NEG-OA materials in solution were able to form aggregates such as spherical or worm-like micelles.

\section{Optical properties of HA-FA-Pg and HA-FA-NEG-OA derivatives}

The absorption/emission features of HA-FA-Pg and HA-FANEG-OA derivatives were characterized in comparison with those of model compounds $\mathbf{4 - 6 ^ { 2 4 }}$ and FA-NEG-OA containing the ferulate fluorophore (Table 3).

The absorption/emission features of ferulic acid have been previously demonstrated to be affected by solvent polarity and pH. In fact, FA possesses in its structure two acidic moiety showing well distinct $\mathrm{p} K_{\mathrm{a}}$ values (4.4 and 9.0, see ref. 5). The neutral FA molecule is barely fluorescent, whereas the singlyionized form is twofold more fluorescent (excitation maximum at $290-310 \mathrm{~nm}$ and emission maximum around 420 $\mathrm{nm}$ ), and the doubly-ionized one is the most emissive specie (at $\mathrm{pH}=10$, the excitation maximum was shifted to $345 \mathrm{~nm}$ and emission maximum to $470 \mathrm{~nm}$, see ref. 5). Furthermore, the decrease in solvent polarity was reported to produce decreases in the excitation, emission, and Stokes shift of FA solutions (i.e. the blue-green fluorescence was quenched by $80 \%$ in chloroform with respect to water, see ref. 5).

By the propargylation of the phenolic $\mathrm{OH}$ of $\mathbf{F A}$ as in compound $\mathbf{6}$, the less acidic group of $\mathbf{F A}$ is lost, and compound 6 shows absorption maxima similar to those reported for FA and slightly sensitive to solvent polarity (methanol: 235, 290 and $318 \mathrm{~nm}$ Fig. 10; dichloromethane: 238, 295 and $324 \mathrm{~nm}$, see ESI $†$ ). On the other hand, the corresponding ester 4, lacking both the ionisable groups, shows very similar absorption spectra in the solvents with well-distinct polarities such as dichloromethane and methanol, with bands peaked at 238, 292 and $319 \mathrm{~nm}$ (see Fig. 10 and ESI $\dagger$ ).

The molar extinction coefficient of ester 4 (i.e. $21817 \mathrm{~L}$ $\mathrm{mol}^{-1} \mathrm{~cm}^{-1}$ ) was found to be well related to the one described for soyamide ferulate (i.e. $19705 \mathrm{~L} \mathrm{~mol}^{-1} \mathrm{~cm}^{-1}$ ). ${ }^{32}$

Ester 4 and the corresponding acid 6 showed similar emissive features in solution with PL maxima centred at $410 \mathrm{~nm}$ and $406 \mathrm{~nm}$ in methanol for $\mathbf{4}$ and $\mathbf{6}$, respectively, while the spectrum shifted at $388 \mathrm{~nm}$ for $\mathbf{4}$ in dichloromethane, in agreement

Table 3 Photophysical properties of 4-6, FA-NEG-OA, and polymeric materials HA-FA-Pg and HA-FA-NEG-OA

\begin{tabular}{|c|c|c|c|c|c|}
\hline \multirow[b]{2}{*}{ Compd } & \multicolumn{3}{|l|}{ Solution $^{a, b, c}$} & \multicolumn{2}{|l|}{ Solid $^{d, e}$} \\
\hline & $\lambda_{\mathrm{ab}}(\mathrm{nm})$ & $\lambda_{\mathrm{em}}(\mathrm{nm})$ & PLQY (\%) & $\lambda_{\mathrm{em}}(\mathrm{nm})$ & PLQY (\%) \\
\hline 6 & $290,320^{a, b}$ & $405^{b}$ & $0.34^{b}$ & $430^{e}\left(450^{d}\right)$ & $<0.1$ \\
\hline 4 & $290,320^{a, b}$ & $390^{a}\left(410^{b}\right)$ & $0.24^{a}\left(0.34^{b}\right)$ & $370^{e}\left(390^{d}\right)$ & 4.4 \\
\hline FA-NEG-OA & $290,320^{a}$ & $400^{a}$ & $0.28^{a}$ & & \\
\hline 5 & $290,320^{a}$ & $390^{a}$ & $0.24^{a}$ & $400^{e}$ & $<0.1$ \\
\hline HA-FA-Pg-2F & $290,320^{c}$ & $450^{c}$ & $0.68^{c}$ & $420^{e}$ & 7.6 \\
\hline HA-FA-Pg-3F & $290,320^{c}$ & $445^{c}$ & $0.75^{c}$ & $420^{e}$ & 5.5 \\
\hline HA-FA-Pg-4F & $290,320^{c}$ & $445^{c}$ & $0.83^{c}$ & $420^{e}$ & 7.3 \\
\hline HA-FA-NEG-OA-4 & $290,320^{c}$ & $440^{c}$ & $0.79^{c}$ & $420^{e}$ & 2.9 \\
\hline HA-FA-NEG-OA-8 & $290,320^{c}$ & $430^{c}$ & $0.87^{c}$ & $415^{e}$ & 4.8 \\
\hline
\end{tabular}

${ }^{a}$ Dichloromethane. ${ }^{b}$ Methanol. ${ }^{c}$ Water. ${ }^{d}$ Spin. ${ }^{e}$ Cast. 
<smiles>C#CCOc1ccc(/C=C/C(=O)OC)cc1OC</smiles><smiles>C#CCOc1ccc(/C=C/C(=O)O)cc1OC</smiles>
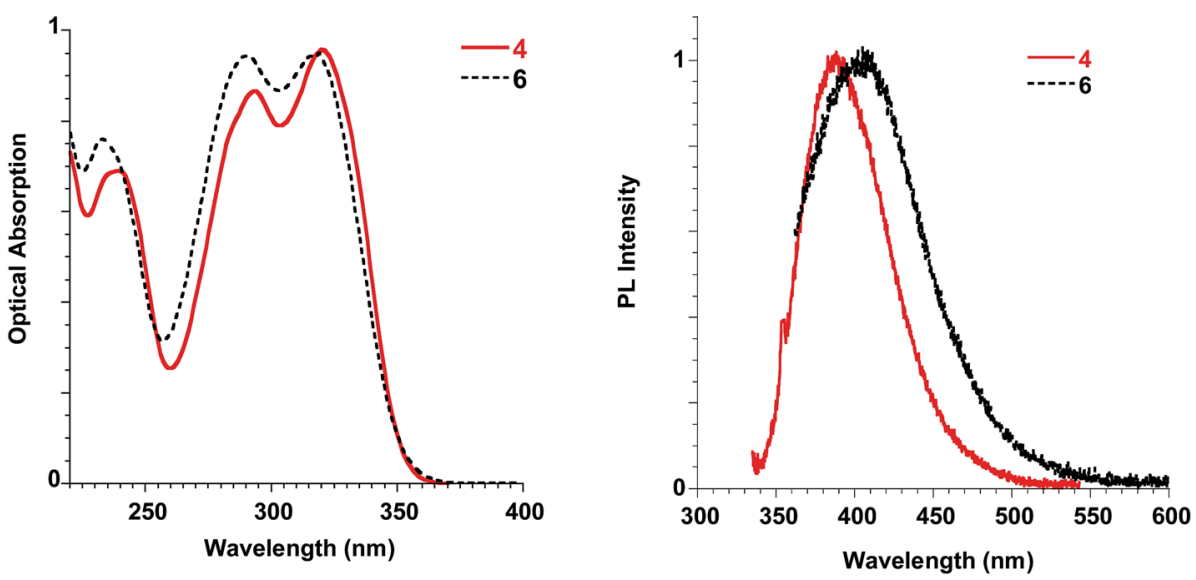

Fig. 10 Absorption (left) and emission (right) spectra of ester 4 in dichloromethane and acid 6 in methanol at $10^{-5} \mathrm{M}$.

with a reduction of the Stokes shift in solvents with lower polarities, as already reported for FA solutions. They were weakly emissive in solution (PLQY $=0.24 \%$ for ester 4 in dichloromethane and $0.34 \%$ for both ester 4 and acid 6 in methanol), but a significant increase in the PLQY (i.e. $4.4 \%$ ) was observed in the films obtained with ester 4 while the emission of acid 6 in the solid state is very low (see Table 3, Fig. 11).

In order to better understand the emissive properties of the two compounds 4 and $\mathbf{6}$ we cooled their diluted solutions down to $77 \mathrm{~K}$ in order to block the molecular motions often responsible for the lack of emission of aggregation-induced emission (AIE) molecules in solution. ${ }^{33,34}$ We observed, for both compounds 4 and 6, a strong increase (a factor of about 50-80) in the emission intensity when the solutions are frozen at $77 \mathrm{~K}$ (see ESI $\dagger$ ). This result demonstrates that, by restricting their molecular motions, both 4 and 6 compounds emit strongly,

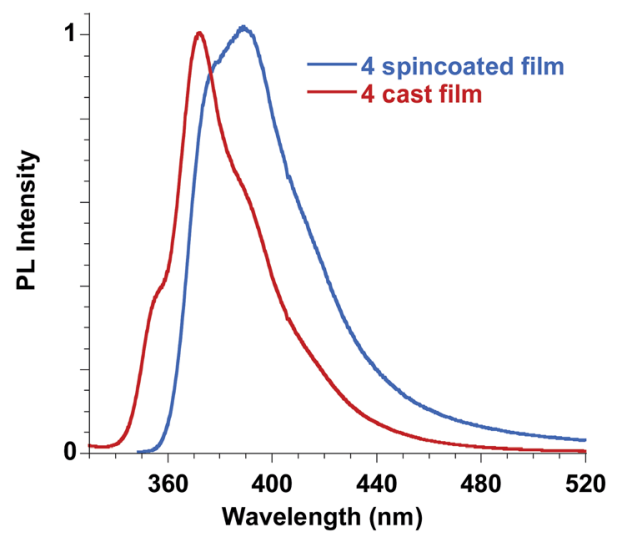

Fig. 11 Emission spectra of ester 4 in the solid state, film obtained by spin coating compared to the film obtained by casting. while upon aggregation only compound $\mathbf{4}$ displays a sensitive emission enhancement. This fact evidences the importance of a proper molecular packing for the exploitation of the AIE properties of this class of fluorogens, as observed for some closely related cinnamic derivatives. ${ }^{35}$ Therefore, compound 4 showed a typical AIE behavior, ${ }^{36,37}$ with photoluminescence quantum yields in the solid state increased by one order of magnitude with respect to the corresponding values measured in solution. Furthermore, we note that the emission spectra in the solid state depend on the film deposition procedure. When the film is obtained by casting the solution a better organization of the molecules is usually obtained with respect to the spincoating deposition, which causes a rapid evaporation of the solvent. As a result, the emission of the cast film displays a sharper and better structured spectrum while that of the spincoated film is closer to the solution one.

This result stimulated our interest in evaluating the effects of the introduction of ferulate fluorogen in the rigid HA backbone as in HA-FA-Pg graft copolymers. The photophysical features of three HA-FA-Pg samples showing different grafting degrees were investigated both in the water solutions and in the solid state. The absorption spectra of the polymeric materials were dominated by the absorption of the ferulate fluorophores with maxima perfectly matching with those of ester 4 (290 and 320 $\mathrm{nm}$ ), whereas a significant red shift was observed in the emission spectra recorded with the water solutions of HA-FA-Pg, as expected due to the high polarity of the solvent (Table 3, Fig. 12).

The PLQY values measured with the water solutions of HAFA-Pg samples resulted higher (in the range of $0.68-0.83 \%$ ) than of the corresponding value obtained with the solution of ester 4 in dichloromethane $(0.24 \%)$. This effect was retained also when HA-FA-NEG-OA were compared with the corresponding model compounds 5 and FA-NEG-OA and could be 


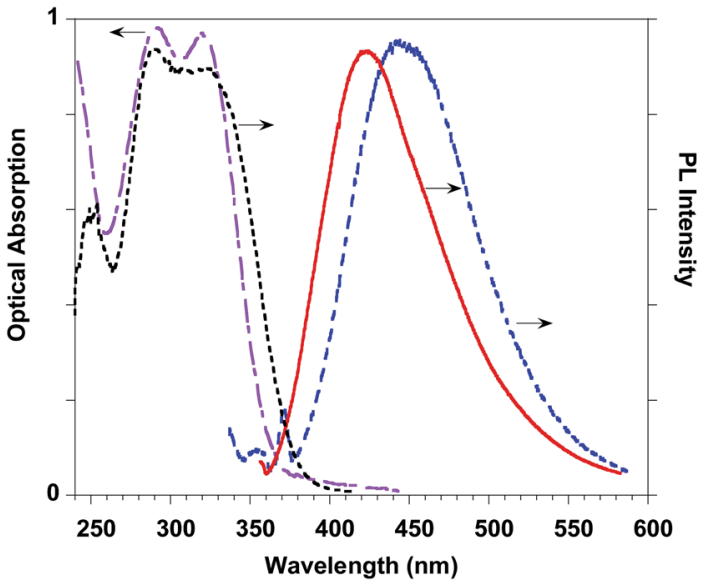

Fig. 12 HA-FA-Pg-3F optical properties: absorption (pink dasheddotted line) and emission (blue dashed line) spectra in water solution. $\mathrm{PL}$ (red line) and PL excitation (black dotted line) spectra of the solid state cast film.

rationalized with the decrease of the mobility of the ferulate fluorogen as suggested by NMR studies.

A further significant increase in the PLQY was observed in the solid-state samples (i.e. cast films) of the polymeric materials, which showed PLQY values (in the range 2.9-7.6\%) sufficiently promising for a further characterization of these materials in view of their potential biomedical applications.

Table 4 Mean particle size, polydispersity index, and surface charge of pure and HA-FA-NEG-OA-8 loaded liposomes obtained by extrusion through $100 \mathrm{~nm}$ polycarbonate membranes

Liposome composition $\quad$ Size $\pm \mathrm{SD}(\mathrm{nm}) \quad$ PDI $\quad \zeta$ potential $\pm \mathrm{SD}(\mathrm{mV})$

$\begin{array}{llll}\mathrm{DOPC} / \mathrm{DOPE} & 105.7 \pm 11.0 & 0.16 & -13.6 \pm 4.8\end{array}$

$\mathrm{DOPC} / \mathrm{DOPE}+\quad 143.9 \pm 6.8 \quad 0.18-41.0 \pm 10.3$

HA-FA-NEG-OA-8

\section{Interactions of HA-FA-NEG-OA derivatives with phospholipid} bilayers

Owing to their peculiar architecture, HA-FA-NEG-OA derivatives were assumed to interact with cell membranes. In order to evaluate this hypothesis, preliminary studies were performed by using unilamellar liposomes as simplified models of cell membranes. Liposomes were synthesized with 1,2-dioleoyl-sn-glycerophosphocholine (DOPC) and 1,2-dioleoyl-sn-glycerophosphoethanolamine (DOPE), which are commonly used in zwitterionic liposomes, ${ }^{38,39}$ since they form a fluid bilayer at room temperature. Furthermore, the phosphocholine and phosphoethanolamine are the most abundant polar head types in the outer cell membrane. ${ }^{\mathbf{4 0}}$

Unilamellar vesicles showing sub-micrometer dimensions (SUV) were prepared by standard procedures, and the mean diameters, polydispersity indexes and $\zeta$-potential of the DOPC/ DOPE liposomes with and without HA-FA-NEG-OA-8 (Table 4) indicated that insertion of the material into liposomes led to an increase in the mean diameter of vesicles. The vesicle diameter changed significantly after the addition (143.9 $\pm 6.8 \mathrm{~nm})$. The low polydispersity indexes of plain and loaded systems revealed that liposomes were not substantially altered by interaction with HA-FA-NEG-OA-8. Indeed, synthesized liposomes remained fairly monodispersed.

Plain DOPC/DOPE liposomes had a small negative $\zeta$-potential, though the net polar head charge of zwitterionic phospholipids is zero. The insertion of HA-FA-NEG-OA-8 changes the zeta potential to more negative values $(-41.0 \mathrm{mV})$ suggesting that the system is chemically stable and no aggregation or flocculation processes may occur in the solution.

Thus, the photophysical characterization of HA-FA-NEGOA-8 derivative was deepened in the phospholipid environment, both in the SUV dispersions in water and in the solid state of the cast films. The results (see ESI $\dagger$ ) suggested that the interaction of HA-FA-NEG-OA-8 derivative with phospholipid bilayers produced negligible effects in the photophysical features of HA-FA-NEG-OA derivatives maintaining the AIE properties of the fluorogen.

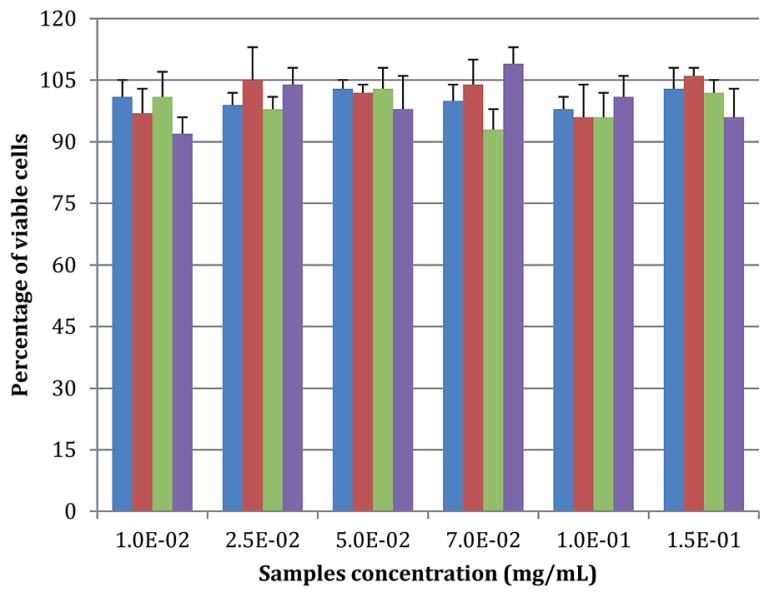

- HA-FA-NEG-OA-4-NIH3T3

- HA-FA-NEG-OA-4-HC

- HA-FA-NEG-OA-8-NIH3T3

- HA-FA-NEG-OA-8-HC

Fig. $13 \mathrm{NIH} 3 \mathrm{~T} 3$ and $\mathrm{HC}$ viability after $24 \mathrm{~h}$ of contact with different concentrations of HA-FA-NEG-OA-4 and HA-FA-NEG-OA-8 materials as determined by the neutral red uptake. Data are mean \pm SD of six replicates. Values are not statistically different versus negative control (complete medium). 


\section{In vitro cytotoxicity and cytocompatibility: cell viability}

Non-confluent adhered fibroblasts and chondrocytes were incubated with different concentrations of HA-FA-NEG-OA materials. Cells were analysed after 24 hours and the results are reported in Fig. 13. The presence of both HA-FA-NEG-OA materials, at any of the tested concentrations, did not affect NIH3T3 and HC viability in comparison to the control (culture medium) and the samples met requirements of ISO 10993-5 standard for cytocompatibility of materials, i.e., cells cultured in the presence of HA-FA-NEG-OA derivatives possessed over $70 \%$ of viability of cells cultured in the standard medium.

Moreover, the results obtained demonstrated the high degree of in vitro cytocompatibility of the test materials which did not interfere with cell viability of both immortalized cells, such as 3T3 fibroblasts, and primary human cells, such as chondrocytes.

\section{Fluorescence imaging}

The internalization of HA-FA-NEG-OA-4 derivative by NIH3T3 cells was evaluated in preliminary studies by fluorescent microscopy. Analysis of fluorescence signal from the NIH3T3 fibroblasts cultured in medium containing HA-FA-NEG-OA-4 material revealed a marked fluorescence distributed in the cells cytoplasm (Fig. 14). These results together with analysis of cell viability data, shows that in the case of both immortalized and primary cells, the presence of HA-FA-NEG-OA-4 do not affect cell growth.

Moreover, the internalization of both the polymeric materials (i.e. HA-FA-NEG-OA-4 and HA-FA-NEG-OA-8) was further confirmed by confocal microscopy in PANC-1 cells with

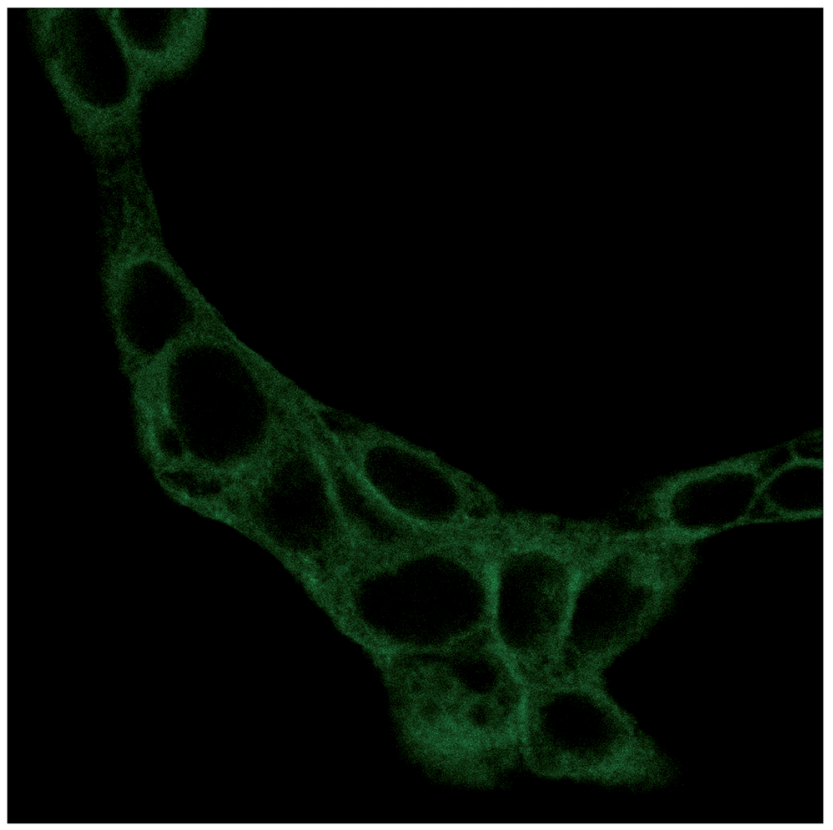

Fig. 14 Fluorescence image of NIH3T3 fibroblast after 15 min of contact with HA-FA-NEG-OA-4 material. Cells were cultured on glass coverslips and imaged on the same slides previous treatment with $4 \%$ paraformaldehyde.

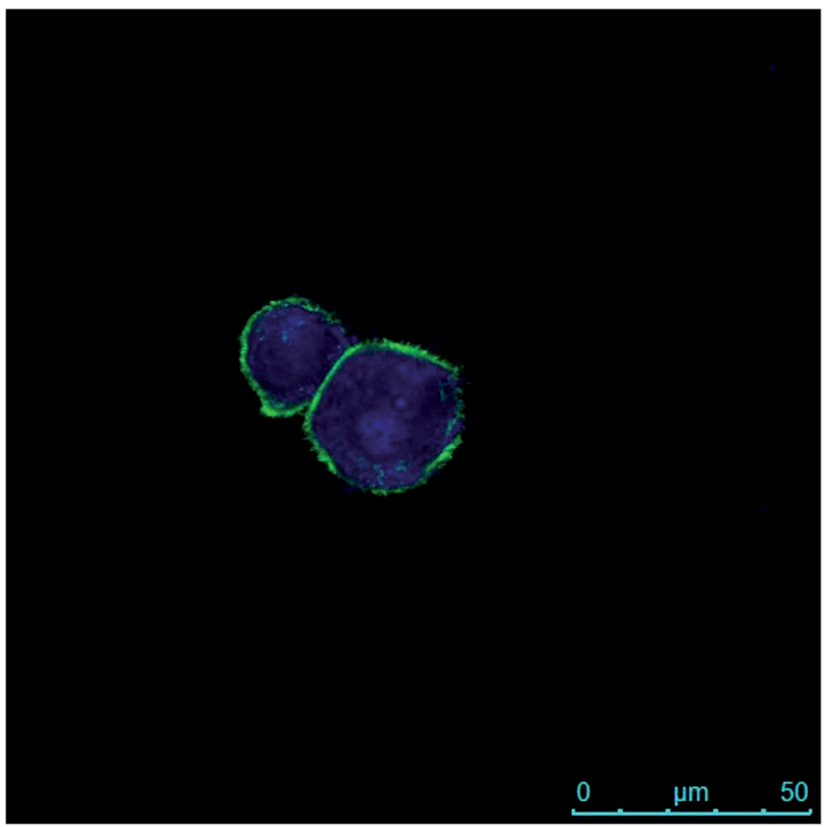

Fig. 15 Fluorescence image of PANC-1 cells after 30 min of contact with HA-FA-NEG-OA-8 material and after $2 \mathrm{~h}$ internalization. The green fluorescence is due to the FITC-conjugated lectin interacting with membranes, whereas the blue emission is produced by HA-FANEG-OA-8 material internalized into the cytoplasm.

membrane labelling by means of FITC-conjugated lectin in order to better visualize the cellular surface (Fig. 15).

In fact, a clear fluorescence signal was observed in the cytoplasm of the cells treated with HA-FA-NEG-OA-4 or HAFA-NEG-OA-8 materials that was attributed to ferulic fluorogen internalized into PANC-1 cells after $30 \mathrm{~min}$ of contact and $2 \mathrm{~h}$ of internalization. This result suggests for HA-FA-NEG-OA materials potential applications in intracellular delivery.

\section{Conclusions}

In conclusion, we have developed a technology platform based on two natural compounds from biorenewable resources, namely low molecular weight HA playing the role of the macromolecular carrier, and FA playing the role of the aggregation-induced emission (AIE) fluorophore bearing clickable propargyl groups. Thus, a short series of hyaluronan-based graft copolymers (HA-FA-Pg) were designed and synthesized to show different grafting degree values and their optical properties were characterized in comparison with reference compounds containing the same ferulate fluorophore. Interestingly, these studies showed that the push-pull structure of the cinnamic scaffold of ferulate fluorophore was quite sensitive to the restriction of intramolecular motions (RIM) phenomenon, thus showing fluorogenic properties. In fact, model compound $\mathbf{4}$ showed a typical aggregation-induced emission behavior, and the introduction of ferulate fluorogen in the rigid HA backbone as in HA-FA-Pg graft copolymers led to PLQY values in the solutions higher than of the 
corresponding value obtained with the solution of model compound 4 in dichloromethane.

In the attempt to demonstrate the usefulness of the technology platform, the propargyl groups of HA-FA-Pg derivatives were exploited in the coupling with a third natural component from biorenewable resources (i.e. oleic acid) through a biocompatible spacer composed of nine monomeric units of ethylene glycol. The resulting HA-FA-NEG-OA derivatives showed selfassembling capabilities in aqueous environment and for this reason were characterized as potential biocompatible selfassembled aggregate forming material for biomedical applications. In particular, HA-FA-NEG-OA derivatives were demonstrated to interact with phospholipid bilayers both in liposomes and living cells retaining the fluorogenic properties and showing a high degree of cytocompatibility.

On the basis of its high modularity, we envisioned for this technology platform a broad applicability. In fact, a broad range of polymeric carriers can be used beside to different fatty acid residues and oligo(ethylene glycol) spacers, with ferulate playing the central role of natural small molecule fluorogenic clickable linker.

\section{Conflicts of interest}

The authors declare no competing financial interest.

\section{Acknowledgements}

Thanks are due to Italian MIUR (Ministero dell'Istruzione, dell'Università e della Ricerca) for financial support.

\section{References}

1 M. M. d. O. Buanafina, Mol. Plant, 2009, 5, 861-872 and references cited therein.

2 J. H. Grabber, J. H. Ralph and R. D. Hatfield, J. Agric. Food Chem., 2000, 48, 6106-6113.

3 G. Wallace and S. Fry, Int. Rev. Cytol., 1994, 151, 229-267.

4 S. Mathew and T. E. Abraham, Crit. Rev. Biotechnol., 2004, 24, 59-83.

5 S. Meyer, A. Cartelat, I. Moya and Z. G. Cerovic, J. Exp. Bot., 2003, 54, 757-769.

6 E. Bramanti, L. Fulgentini, R. Bizzarri, F. Lenci and A. Sgarbossa, J. Phys. Chem. B, 2013, 117, 13816-13821.

7 A. Noel, Y. P. Borguet, J. E. Raymond and K. L. Wooley, Macromolecules, 2014, 47, 2974-2983.

8 N. L. Morozowich, J. L. Nichol, R. J. Mondschein and H. R. Allcock, Polym. Chem., 2012, 3, 778-786.

9 N. L. Morozowich, J. L. Nichol and H. R. Allcock, Chem. Mater., 2012, 24, 3500-3509.

10 S. A. Miller, ACS Macro Lett., 2013, 2, 550-554.

11 H.-G. Elias and J. A. Palacios, Makromol. Chem., 1985, 186, 1027-1045.

12 J. Du, Y. Fang and Y. Zheng, Polymer, 2007, 48, 5541-5547.

13 M. F. Beristain, M. Nakamura, K. Nagai and T. Ogaw, Des. Monomers Polym., 2009, 12, 257-263.
14 S. Kanehashi, T. Nagasawa, M. Kobayashi, S. L. Lee, M. Nakamura, S. Sato, M. F. Beristain, T. Ogawa, T. Miyakoshi and K. Nagai, J. Appl. Polym. Sci., 2013, 130, 277-286.

15 F. Puoci, F. Iemma, M. Curcio, O. I. Parisi, G. Cirillo, U. G. Spizzirri and N. Picci, J. Agric. Food Chem., 2008, 56, 10646-10650.

16 E. A. Castillo, H. Miura, M. Hasegawa and T. Ogawa, Des. Monomers Polym., 2004, 7, 711-725.

17 J. Quian and B. Z. Tang, Chem, 2017, 3, 56-91.

18 M. Cohen, D. Joester, B. Geiger and L. Addadi, ChemBioChem, 2004, 5, 1393-1399.

19 Y. Zhong, K. Goltsche, L. Cheng, F. Xie, F. Meng, C. Deng, Z. Zhong and Z. Haag, Biomaterials, 2016, 84, 250-261.

20 F. Dosio, S. Arpicco, B. Stella and E. Fattal, Adv. Drug Delivery Rev., 2016, 97, 204-236.

21 V. Orian-Rousseau, Eur. J. Cancer, 2010, 46, 1271-1277.

22 A. Cappelli, G. Grisci, M. Paolino, G. Giuliani, A. Donati, R. Mendichi, R. Artusi, M. Demiranda, A. Zanardi, G. Giorgi and S. Vomero, J. Mater. Chem. B, 2014, 2, 4489-4499.

23 G. Valacchi, G. Grisci, C. Sticozzi, Y. Lim, M. Paolino, G. Giuliani, R. Mendichi, G. Belmonte, R. Artusi, A. Zanardi, P. Garofalo, G. Giorgi, A. Cappelli and L. Rovati, J. Mater. Chem. B, 2015, 3, 7037-7045.

24 A. Cappelli, M. Paolino, G. Grisci, V. Razzano, G. Giuliani, A. Donati, C. Bonechi, R. Mendichi, S. Battiato, F. Samperi, C. Scialabba, G. Giammona, F. Makovec and M. Licciardi, Polym. Chem., 2016, 7, 6529-6544.

25 M. Licciardi, C. Scialabba, G. Giammona, M. Paolino, V. Razzano, G. Grisci, G. Giuliani, F. Makovec and A. Cappelli, J. Nanopart. Res., 2017, 19, 197-207.

26 R. Mendichi and A. Giacometti Schieroni, in Current Trends in Polymer Science, ed. S. G. Pandalai, Trans-World Research Network: Trivandrum, India, 2001, vol. 6, pp. 17-32.

27 P. J. Wyatt, Anal. Chim. Acta, 1993, 272, 1-40.

28 J. Moreau, U. Giovanella, J.-P. Bombenger, W. Porzio, V. Vohra, L. Spadacini, G. Di Silvestro, L. Barba, G. Arrighetti, S. Destri, M. Pasini, M. Saba, F. Quochi, A. Mura, G. Bongiovanni, M. Fiorini, M. Uslenghi and C. Botta, ChemPhysChem, 2009, 10, 647-653.

29 ISO 10995-5:2009, Biological evaluation of medical devices Part 5: Tests for cytotoxicity: in vitro methods.

30 M. H. Levitt, Spin Dynamics: Basics of Nuclear Magnetic Resonance, John Wiley \&Sons, 2001.

31 E. H. M. Sakho, E. Allahyari, O. S. Oluwafemi, S. Thomas and N. Kalarikkal, Chapter 2 - Dynamic Light Scattering (DLS), Micro and Nano Technologies, in Thermal and Rheological Measurement Techniques for Nanomaterials Characterization, ed. S. Thomas, R. Thomas, A. K. Zachariah and R. K. Mishra, Amsterdam, Netherlands, 2017, pp. 37-49.

32 J. W. Rawlins, M. Pramanik and S. K. Mendon, J. Am. Oil Chem. Soc., 2008, 85, 783-789.

33 Y. N. Hong, J. W. Y. Lam and B. Z. Tang, Chem. Soc. Rev., 2011, 40, 5361.

34 E. Cariati, V. Lanzeni, E. Tordin, R. Ugo, C. Botta, A. Giacometti Schieroni, A. Sironi and D. Pasini, Phys. Chem. Chem. Phys., 2011, 13, 18005. 
35 V. Razzano, M. Paolino, A. Reale, G. Giuliani, R. Artusi, G. Caselli, G. Visintin, F. Makovec, A. Donati, F. Villafiorita-Monteleone, C. Botta and A. Cappelli, ACS Omega, 2017, 2, 5453-5459.

36 J. Mei, N. L. C. Leung, R. T. K. Kwok, J. W. Y. Lam and B. Z. Tang, Chem. Rev., 2015, 115, 11718-11940.

37 V. Sathish, A. Ramdass, P. Thanasekaran, K.-L. Lu and S. Rajagopal, J. Photochem. Photobiol., C, 2015, 23, 25-44.
38 C. Della Giovampaola, A. Capone, L. Ermini, P. Lupetti, E. Vannuccini, F. Finetti, S. Donnini, M. Ziche, A. Magnani, G. Leone, C. Rossi, F. Rosati and C. Bonechi, Biochim. Biophys. Acta, 2017, 1861(4), 860-870.

39 E. Moretti, L. Mazzi, C. Bonechi, M. C. Salvatici, F. Iacoponi, C. Rossi and G. Collodel, Reprod. Toxicol., 2016, 60, 140-147. 40 The Structure of Biological Membranes, ed. P. Yeagle, CRC Press, Boca Raton, Florida, 2005. 